\title{
The IACOB project
}

\section{New observational clues to understand macroturbulent broadening in massive 0 - and B-type stars ${ }^{\star}$}

\author{
S. Simón-Díaz ${ }^{1,2}$, M. Godart ${ }^{1,2,3}$, N. Castro ${ }^{4,5}$, A. Herrero ${ }^{1,2}$, C. Aerts ${ }^{6,7}$, J. Puls ${ }^{8}$, J. Telting ${ }^{9}$, and L. Grassitelli ${ }^{4}$ \\ 1 Instituto de Astrofísica de Canarias, 38200 La Laguna, Tenerife, Spain \\ e-mail: ssimon@iac.es \\ 2 Departamento de Astrofísica, Universidad de La Laguna, 38205 La Laguna, Tenerife, Spain \\ 3 Institut d'Astrophysique et de Géophysique, Université de Liège, 17 allée du 6 Août, 4000 Liège, Belgium \\ 4 Argelander Institut für Astronomie, Auf den Hägel 71, 53121 Bonn, Germany \\ 5 Department of Astronomy, University of Michigan, 1085 S. University Avenue, Ann Arbor, MI 48109-1107, USA \\ ${ }^{6}$ Instituut voor Sterrenkunde, KU Leuven, Celestijnenlaan 200D, 3001 Leuven, Belgium \\ 7 Department of Astrophysics/IMAPP, Radboud University Nijmegen, 6500 GL Nijmegen, The Netherlands \\ 8 LMU Munich, Universitäts-Sternwarte, Scheinerstr. 1, 81679 München, Germany \\ 9 Nordic Optical Telescope, Rambla José Ana Fernández Pérez 7, 38711 Breña Baja, Spain
}

Received 17 March 2016 / Accepted 16 August 2016

\begin{abstract}
Context. The term macroturbulent broadening is commonly used to refer to a certain type of non-rotational broadening affecting the spectral line profiles of O- and B-type stars. It has been proposed to be a spectroscopic signature of the presence of stellar oscillations; however, we still lack a definitive confirmation of this hypothesis.

Aims. We aim to provide new empirical clues about macroturbulent spectral line broadening in O- and B-type stars to evaluate its physical origin.

Methods. We used high-resolution spectra of 430 stars with spectral types in the range O4-B9 (all luminosity classes) compiled in the framework of the IACOB project. We characterized the line broadening of adequate diagnostic metal lines using a combined Fourier transform and goodness-of-fit technique. We performed a quantitative spectroscopic analysis of the whole sample using automatic tools coupled with a huge grid of FASTWIND models to determine their effective temperatures and gravities. We also incorporated quantitative information about line asymmetries into our observational description of the characteristics of the line profiles, and performed a comparison of the shape and type of line-profile variability found in a small sample of $\mathrm{O}$ stars and $\mathrm{B}$ supergiants with still undefined pulsational properties and B main-sequence stars with variable line profiles owing to a well-identified type of stellar oscillations or to the presence of spots in the stellar surface.

Results. We present a homogeneous and statistically significant overview of the (single snapshot) line-broadening properties of stars in the whole $\mathrm{O}$ and $\mathrm{B}$ star domain. We find empirical evidence of the existence of various types of non-rotational broadening agents acting in the realm of massive stars. Even though all these additional sources of line-broadening could be quoted and quantified as a macroturbulent broadening from a practical point of view, their physical origin can be different. Contrarily to the early- to late-B dwarfs and giants, which present a mixture of cases in terms of line-profile shape and variability, the whole O-type and B supergiant domain (or, roughly speaking, stars with $M_{\text {ZAMS }} \gtrsim 15 M_{\odot}$ ) is fully dominated by stars with a remarkable non-rotational broadening component and very similar profiles (including type of variability). We provide some examples illustrating how this observational dataset can be used to evaluate scenarios aimed at explaining the existence of sources of non-rotational broadening in massive stars.
\end{abstract}

Key words. stars: early-type - stars: fundamental parameters - stars: massive - stars: rotation - stars: oscillations techniques: spectroscopic

\section{Introduction}

Line profiles in optical spectra of $\mathrm{OB}$ stars $^{1}$ are not only broadened by rotation. This statement has been known since

\footnotetext{
* Full Table 1 is only available at the CDS via anonymous ftp to cdsarc.u-strasbg.fr (130.79.128.5) or via

http://cdsarc.u-strasbg.fr/viz-bin/qcat?]/A+A/597/A22

1 Throughout this paper, and following Reed (2003), we use the term OB stars to refer to O- and early-B type stars on the main sequence and their evolved descendants, the B supergiants. The remaining B-type stars (dwarfs and giants) are considered as a separate group.
}

the first large spectroscopic surveys of massive stars in the late 1950s (Slettebak 1956; Conti \& Ebbets 1977). First indirect suspicions, supported by the absence of narrow-line stars of this type, were soon confirmed with the advent of high-resolution spectrographs: the $\mathrm{V}$ shape of some of the profiles did not correspond to an exclusively rotationally broadened line. More recently, the use of Fourier transform techniques (cf. Gray 1976), in combination with profile fitting techniques, has allowed us to have access to actual projected rotational velocities $(v \sin i)$ in OB stars and quantify the relative contribution of the non-rotational and rotational 
broadenings (e.g., Simón-Díaz \& Herrero 2007, 2014, and references therein).

The extra source of line broadening found in OB stars has been commonly quoted as macroturbulent broadening (and quantified as a macroturbulent velocity, $v_{\mathrm{mac}}$ ). However, its connection with large-scale turbulent motions of material in the line-formation region ${ }^{2}$ is highly improbable (see, e.g., Simón-Díaz et al. 2010). An alternative scenario proposed for the $\mathrm{O}$ and $\mathrm{B}$ star domain relates the above mentioned extra broadening to the effect of stellar oscillations on the line profiles (e.g., Lucy 1976; Howarth 2004; Aerts et al. 2009). In this context, not only the most commonly considered heat-driven non-radial modes, but also other spectroscopic variability phenomena identified and/or predicted in massive stars may play a role (e.g., rotational modulation, strange modes, stochastically excited non-radial modes and/or convectively driven internal gravity waves).

The presence of a variable pulsational broadening component is well known in observed line profiles of B dwarfs and giants located in the $\beta$-Cep and SPB (slowly pulsating B-type star) instability domains (e.g., Aerts et al. 2014, and references therein). Indirect arguments presented in recent years (Aerts et al. 2009; Simón-Díaz et al. 2010) indicates that this may also be the case for B supergiants (Sgs). However, the macroturbulent-pulsational broadening connection in the whole OB star domain, which is a region of the Hertzsprung-Russel diagram that to-date is (by far) less explored and understood from an asteroseismic point of view, still requires direct (observational) confirmation. Indeed, even if we assume this likely connection, the exact driving mechanism (or mechanisms) of the type of oscillations that might result in the observed (macroturbulent) profiles remains undefined (but see Aerts et al. 2009; Cantiello et al. 2009; Samadi et al. 2010; Shiode et al. 2013; Sundqvist et al. 2013a; Simón-Díaz 2015; Aerts \& Rogers 2015; Grassitelli et al. 2015a).

With the aim of providing a set of empirical constraints that could be used to assess the pulsational (or any other) hypothesis to explain the physical origin of macroturbulent broadening in OB stars, in 2008 we started the compilation of a high-quality spectroscopic database including multi-epoch observations of a large sample of bright $(V<9)$ northern O- and B-type stars. This observational material, which has now become part of the IACOB spectroscopic database (Simón-Díaz et al. 2011a, 2015), presently comprises (a) high-resolution spectra of $\approx 620$ Galactic stars covering spectral types between O4 and B9 and all luminosity classes, and (b) time-series spectra with a time span of several years and various types of time coverage for a selected sample of targets.

In Simón-Díaz et al. (2010) we used part of these observations to present first empirical evidence for the existence of a correlation between the macroturbulent broadening and photospheric line-profile variations in a sample of $13 \mathrm{OB}$ Sgs. In Simón-Díaz \& Herrero (2014) we concentrated on the line-broadening analysis of $\approx 200 \mathrm{O}$ and early-B stars to investigate the impact of other sources of non-rotational broadening on the determination of projected rotational velocities in $\mathrm{OB}$ stars. In this paper we benefit from a much larger and extended (in terms of spectral type coverage)

\footnotetext{
2 This concept of macroturbulent broadening was initially introduced and studied in the context of cool stars (see, e.g., the review by Gray 1978, and other historical references therein). Even though the use of this term has been extended to other star domains, it does not necessarily refer to the same type of broadening mechanism or the same physical origin.
}

spectroscopic dataset to provide new observational clues to step forward in our understanding of macroturbulent broadening in massive stars. In particular, with this work we increase and improve the available information about the single snapshot properties of this enigmatic line broadening in the whole $\mathrm{O}$ and $\mathrm{B}$ star domain, which is presently fragmentary and not necessarily homogeneous (e.g., Ryans et al. 2002; Dufton et al. 2006; Lefever et al. 2007, 2010; Fraser et al. 2010; Bouret et al. 2012; Simón-Díaz \& Herrero 2014; Markova et al. 2014; Martins et al. 2015; Mahy et al. 2015).

In this paper, we focus on the global aspects of the line-broadening properties of the large sample we composed. Subsequent work will be tuned toward time-series analysis for a subsample of $\approx 70-100$ targets. Sections 2 and 3 describe the observational dataset and spectroscopic analysis tools we used to extract the level of line-broadening, line-asymmetry, and spectroscopic parameters. The variety of profiles found in the sample is illustrated and discussed in Sect. 4.1, while the distribution of stars in the $v \sin i-v_{\operatorname{mac}}$ and spectroscopic Hertzsprung-Russell (sHR; Langer \& Kudritzki 2014; Castro et al. 2014) diagrams is discussed in Sect. 4.2. An extensive discussion on how these observations, once combined with further information about line-profile skewness and variability, can help us to definitely identify the origin of the various sources of non-rotational broadening found in massive stars, is presented in Sect. 5. Last, a summary of our work, including the main conclusions and some future prospects, are presented in Sect. 6.

\section{Observations}

The main observational sample discussed in this paper comprises high-resolution, single snapshot spectra of 430 Galactic stars. Basically, from the $618 \mathrm{O}$ - and B-type stars in the IACOB spectroscopic database as for November 2015, we discarded:

- 80 stars showing clear signatures of being a double line spectroscopic binary, a multiple system or, more generally, a composite spectrum in at least one of the IACOB spectra;

- 25 stars in which all the main diagnostic lines considered to obtain information about $v \sin i$ and $v_{\text {mac }}$ (see Sect. 3.1) are weak, absent or present strong spectroscopic peculiarities, among this subsample one can find stars with a spectral type earlier than $\mathrm{O} 4$, very fast rotators and some Oe and Be stars; - 83 stars having a projected rotational velocity larger than $200 \mathrm{~km} \mathrm{~s}^{-1}$ (see explanation in Sect. 4.2.1).

The IACOB database includes spectra from two different instruments: the FIES (Telting et al. 2014) and HERMES (Raskin et al. 2011) spectrographs attached to the $2.56 \mathrm{~m}$ Nordic Optical Telescope and the $1.2 \mathrm{~m}$ Mercator telescope, respectively. Both instruments provide a complete wavelength coverage between 3800 and $7000 \AA$ (9000 $\AA$ for the case of HERMES spectra), and the associated resolving power (R) of the spectra is 25000, 46000 (FIES) and 85000 (HERMES). By default, all the spectra in the IACOB database are reduced using the corresponding available pipelines (FIEStool ${ }^{3}$ and HermesDRS ${ }^{4}$, respectively) and they are normalized by means of our own procedures implemented in IDL.

\footnotetext{
http://www.not.iac.es/instruments/fies/fiestool/ FIEStool.html

4 http://www . mercator.iac.es/instruments/hermes/ hermesdrs.php
} 
Table 1. Stars considered for this paper, including information about line broadening, stellar parameters, and the quantity $R S k$ (relative skewness, see Eq. (1)).

\begin{tabular}{|c|c|c|c|c|c|c|c|c|c|c|c|}
\hline \multirow[t]{2}{*}{ Target } & \multirow[t]{2}{*}{$\mathrm{SpC}$} & \multirow[t]{2}{*}{ Line } & \multirow[t]{2}{*}{$S / N_{\mathrm{c}}$} & \multirow[t]{2}{*}{$E W$} & \multicolumn{2}{|c|}{$v \sin i$} & \multirow{2}{*}{$\begin{array}{r}v_{\mathrm{mac}} \\
\mathrm{GOF}\end{array}$} & \multirow[t]{2}{*}{$R S k$} & \multirow[t]{2}{*}{$\sigma_{\mathrm{RSk}}$} & \multirow[t]{2}{*}{$\log T_{\text {eff }}$} & \multirow[t]{2}{*}{$\log \mathscr{L} / \mathscr{L}_{\odot}$} \\
\hline & & & & & FT & GOF & & & & & \\
\hline HD 16582 & B2 IV & $\begin{array}{l}\ldots \\
\text { Si III }\end{array}$ & $\ldots$ & $\begin{array}{c}\ldots \\
138\end{array}$ & \% & 9 & $\begin{array}{c}\cdots \\
19\end{array}$ & 0.02 & 0.05 & 4.34 & 2.94 \\
\hline HD 17081 & B7 V & Mg II & 195 & 294 & 18 & 19 & 24 & -0.03 & 0.02 & 4.12 & 2.40 \\
\hline HD 17603 & O7.5 Ib(f) & O III & 239 & 317 & 109 & 99 & 115 & -0.01 & 0.02 & 4.53 & 4.21 \\
\hline HD 17743 & B8 III & Mg II & 157 & 214 & 48 & 47 & 22 & 0.00 & 0.06 & 4.13 & 2.21 \\
\hline HD 18409 & O9.7 Ib & O III & 256 & 179 & 131 & 128 & $<88$ & 0.08 & 0.16 & 4.51 & 3.99 \\
\hline HD 18604 & B6 III & Mg II & 184 & 305 & 131 & 132 & $<50$ & 0.07 & 0.08 & 4.11 & 2.44 \\
\hline HD 19820 & O8.5 III(n)((f)) & O III & 316 & 251 & 144 & 147 & $<54$ & -0.22 & 0.11 & 4.51 & 3.91 \\
\hline$\ldots$ & $\ldots$ & $\ldots$ & $\ldots$ & $\ldots$ & $\ldots$ & $\ldots$ & $\ldots$ & $\ldots$ & $\ldots$ & $\ldots$ & $\cdots$ \\
\hline
\end{tabular}

Notes. The line used to determine the line-broadening parameters is also indicated, along with its equivalent width and the signal-to-noise ratio of the adjacent continuum. Spectral classifications indicated in Col. 2 must be handled with caution since they come from various sources, not all of which are equally reliable. $E W$ in $\mathrm{m} \AA, v \sin i$ and $v_{\text {mac }}$ in $\mathrm{km} \mathrm{s}^{-1}, T_{\text {eff }}$ in $\mathrm{K}$. The full table is available at the CDS.

The best signal-to-noise ratio $(\mathrm{S} / \mathrm{N})$ spectrum per considered star was selected for the purposes of this study. The typical $\mathrm{S} / \mathrm{N}$ is in the range 150-300. In many cases, especially for targets brighter than $V=6$, the same star was observed with both instruments. For these stars, we preferred the HERMES over the FIES spectrum whenever the associated $\mathrm{S} / \mathrm{N}$ is similar because of the larger resolving power.

This main (single snapshot) spectroscopic dataset is complemented with high-resolution spectroscopic time series of a sample of eight selected targets (Sect. 5.2.3). Half of these stars are well-known pulsators and stars with spots located in the B main-sequence star domain (selected from the sample described and analyzed in Aerts et al. 2014, and references therein), the other four stars correspond to bright $\mathrm{O}$ stars and B Sgs surveyed by the IACOB project (as an extension of the observations presented in Simón-Díaz et al. 2010). In all cases we rely on more than 90 spectra gathered during more than four years. These observations are used to provide a first comparison of the type of line-profile variability present in OB stars with an important contribution of the macroturbulent broadening and $\mathrm{B}$ main-sequence stars with well-identified causes of spectroscopic variability.

\section{Tools and methods}

In this section we describe the strategy we followed to extract from the spectra information about (a) $v \sin i$ and $v_{\text {mac }}$; (b) the amount of asymmetry of the diagnostic lines considered to derive the line-broadening parameters; and (c) the stellar parameters that allow us to locate the stars in the spectroscopic HR diagram. The results from the analysis, which is used for the discussion in Sects. 4 and 5, are summarized in Table 1, where the meaning of each column is explained below.

\subsection{Line-broadening parameters}

We applied the iacob-broad tool (Simón-Díaz \& Herrero 2014 , in the following SDH14) to the O III $\lambda 5591$, Si III $\lambda 4552$, Mg II $\lambda 4481$, or C II $\lambda 4267$ line, depending on the spectral type of the star, to derive $v \sin i$ and $v_{\text {mac }}$ in the whole sample. We followed the strategy described in SDH14; namely, the line-broadening analysis is based on a combined Fourier transform (FT) plus goodness-of-fit (GOF) methodology where we consider a radial-tangential definition for the macroturbulent profile, with equal radial and tangential components, and the starting intrinsic profile for the GOF computation is simplified by a $\delta$ function.

In most cases, the outcome of the global rectification applied to all IACOB spectra was fairly good when zooming into the smaller spectral windows associated with the lines to be analyzed. However, whenever necessary, we activated the option available in iacob-broad to locally improve the normalization before performing the line-broadening analysis.

Results from the line-broadening analysis of the 430 stars are indicated in Cols. 6-8 of Table 1 along with some information about the equivalent width (EW, Col. 5) of the considered diagnostic line (Col. 3) and the $\mathrm{S} / \mathrm{N}$ of the adjacent continuum (Col. 4). We provide both FT and GOF solutions for $v \sin i$, but only the latter, together with the associated $v_{\text {mac }}$ are considered in this paper. The main reason for this is that we found a general good agreement between the $v \sin i$ derived by means of both methods (better than $\pm 10 \mathrm{~km} \mathrm{~s}^{-1}$ in most cases; see Fig. 1). But we found that, first, the GOF approach is less affected by the subjectivity in the selection of the first zero of the Fourier transform in some complicated cases and, second, the GOF solution allows us to detect cases in which we can only provide upper limits to $v_{\mathrm{mac}}$. The latter refers to situations in which the $v_{\text {mac }}$ value associated with the best-fitting solution (minimum $\chi^{2}$ ) is higher than zero, but $v_{\text {mac }}=0$ is still an acceptable solution (below the 1- $\sigma$ confidence level). As we show and discuss in Sect. 4, these cases normally correspond to profiles in which the rotational broadening component dominates. Those cases in which $v_{\text {mac }}$ could not be properly determined are quoted in Table 1 by providing the value corresponding to the best-fitting solution as an upper limit.

\subsection{Line-profile asymmetry}

Most of the previous work on macroturbulent broadening in OB stars concentrates on the characterization of the line profiles via $v \sin i$ and $v_{\mathrm{mac}}$. However, as is well known in the context of stellar oscillations (cf. Chapts. 5 and 6 of Aerts et al. 2010b), such a simplistic two-parameter description lacks an important piece of information about the properties of the profiles: the amount of line asymmetry. In this respect, Schrijvers et al. (1997) is a particularly illustrative study in which the predicted variability of line profiles from adiabatic non-radial pulsations in rotating stars is shown for a large variety of oscillation 


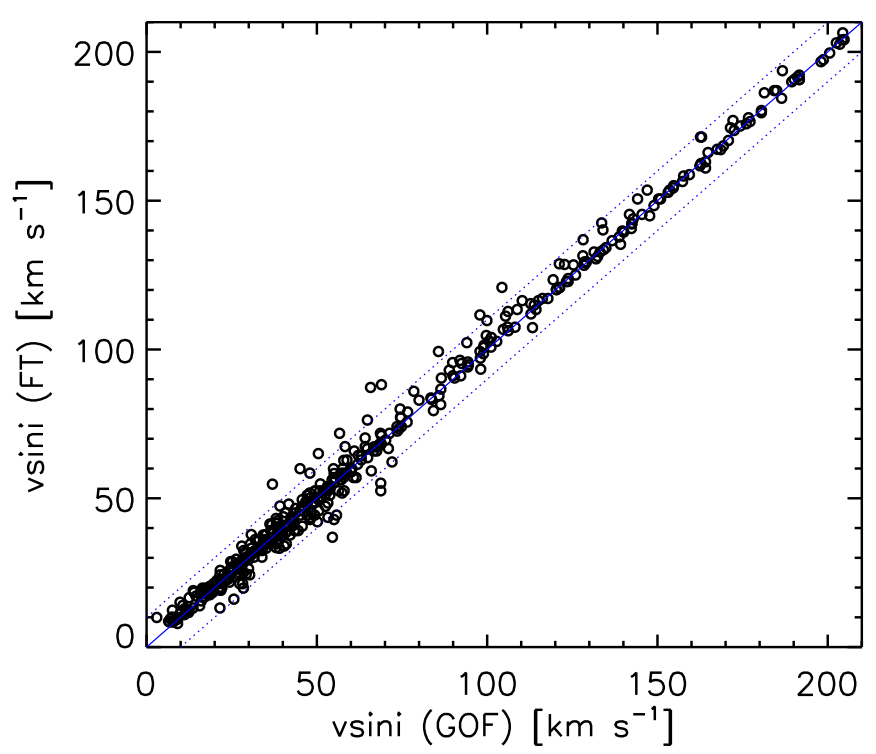

Fig. 1. Comparison of the $v \sin i$ values determined by means of the FT and GOF methodologies using the iacob-broad tool. The agreement is better than $\pm 10 \mathrm{~km} \mathrm{~s}^{-1}$ for $95 \%$ of the sample, and better than $\pm 20 \mathrm{~km} \mathrm{~s}^{-1}$ for the remaining stars.

and rotation parameters. This reference work contains various diagnostics used to describe the snapshot characteristics of the line profiles, a combination of which we adopt here as the relative skewness $(R S k)$. We define this quantity as

$R S k \equiv\left\langle v^{3}\right\rangle /\left\langle v^{2}\right\rangle^{3 / 2}$,

where

$\left\langle v^{n}\right\rangle=\frac{\int_{-\infty}^{\infty}(v-\langle v\rangle)^{n}(1-F(v)) \mathrm{d} v}{\int_{-\infty}^{\infty}(1-F(v)) \mathrm{d} v} \quad$ for $n=2,3$

are the second and third normalized central moments of a spectral line denoted as $(v, F(v))$, adopting the definition used in Schrijvers et al. (1997), and

$\langle v\rangle=\frac{\int_{-\infty}^{\infty} v(1-F(v)) \mathrm{d} v}{\int_{-\infty}^{\infty}(1-F(v)) \mathrm{d} v}$.

The dimensionless quantity defined by Eq. (1) - which, in statistical terms, is quoted as the third standardized moment or (Pearson's) moment coefficient of skewness - represents the amount of skewness of the line profile relative to its total width. This allows us to compare the amount of asymmetry for lines with very different widths in a meaningful way.

We used the same line profiles considered to derive the line-broadening parameters (Sect. 3.1) for the computation of the first three moments and, ultimately, the quantity $R S k$. To compute the latter, we considered various integration limits and found the best overall choice to be an integration centered on $\langle v\rangle$ and extending over $\pm 2.5 \sqrt{\left\langle v^{2}\right\rangle}$. These integration limits were selected as the best compromise between including as much information as possible from the extended wings of the V-shaped line profiles, while avoiding spurious subtleties associated with the noise of the adjacent continuum.

The computed values of $R S k$ for the whole sample of stars, along with their corresponding uncertainties, are presented in Cols. 9 and 10 in Table 1. These uncertainties result from the formal propagation of errors for Eqs. (1)-(3), assuming that the only source of uncertainty is the noise associated with the normalized flux of the line profile, which is inversely proportional to the $\mathrm{S} / \mathrm{N}$ of the adjacent continuum $\left(\mathrm{S} / \mathrm{N}_{\mathrm{c}}\right)$. Given the stability of the spectrographs and the level of precision of the wavelength calibration, this approximation is fully justified.

\subsection{Stellar parameters}

The spectroscopic stellar parameters of the sample were obtained by means of iacob-gbat (Simón-Díaz et al. 2011b, $\mathrm{O}$ stars) or an updated version of the tool described in Castro et al. (2012, B stars). These tools, aimed at performing quantitative spectroscopic analyses of O- and B-type stars in a fast, automatic way, are based on a huge, pre-computed grid of FASTWIND (Santolaya-Rey et al. 1997; Puls et al. 2005; Rivero González et al. 2012) synthetic spectra and a $\chi^{2}$ minimization line-profile fitting technique. Some notes on the followed strategy can be found in Lefever et al. (2007), Castro et al. (2012), and Sabín-Sanjulián et al. (2014). In this case, we fixed $v \sin i$ and $v_{\mathrm{mac}}$ to the values resulting from the line-broadening analysis described above. From the whole set of output parameters provided by the automatic tools, we concentrate on the derived $T_{\text {eff }}$ and $\log g$ because they allow us to locate the studied stars in the sHR diagram.

We provide $\log T_{\text {eff }}$ and $\log \mathscr{L} / \mathscr{L}_{\odot}$ (where $\mathscr{L}:=T_{\text {eff }}{ }^{4} / g$, Langer \& Kudritzki 2014) in the two last columns of Table 1 to facilitate the identification of stars in the corresponding diagrams. In addition, we highlight below some important points regarding the compiled set of stellar parameters:

- Given the quality of the spectroscopic observations and the strategy we have followed for the spectroscopic analysis, we can assume $\approx 5 \%$ and $\approx 0.15$ dex as rough estimations for the uncertainties in the derived $T_{\text {eff }}$ and $\log g$, respectively.

- There are $\approx 50$ stars for which we do not provide stellar parameters in Table 1. Most of these stars are late-B stars whose parameters lay outside our grid of FASTWIND models. The lower $T_{\text {eff }}$ boundary of the grid is $11000 \mathrm{~K}$ and this implies that our analysis tools do not provide reliable parameters for stars with $T_{\text {eff }} \leq 12000 \mathrm{~K}$.

- We have not checked one-by-one all the analyzed spectra in detail. Therefore, the stellar parameters quoted in Table 1 must be considered with caution for purposes other than those discussed in this paper, especially concerning the investigation of individual stars.

\section{Results}

\subsection{Line profiles in $O$ and $B$ stars: a qualitative overview}

Figures 2 and 3 show some representative examples of the various types of line profiles found in stars in the IACOB sample. In all cases the observed profiles, colored and labeled following the guidelines described in Sect. 4.2.1, are complemented with information from the outcome of the line-broadening and line-asymmetry analyses (see Sect. 3). We also overplot, for guidance purposes, synthetic profiles with the same equivalent width as the observed profiles, convolved with the indicated $v \sin i$ (dotted lines) and $v \sin i+v_{\text {mac }}$ values (dashed lines). The comparison between the dotted lines and the observed profiles allows us to visualize the effect of the non-rotational broadening on the shape of the lines for different $v \sin i-v_{\text {mac }}$ combinations. The dashed lines can be used to assess the quality of the final fit and visually identify line profiles that are clearly asymmetric or have a bumpy shape (see, e.g., Fig. 3 and description below). 

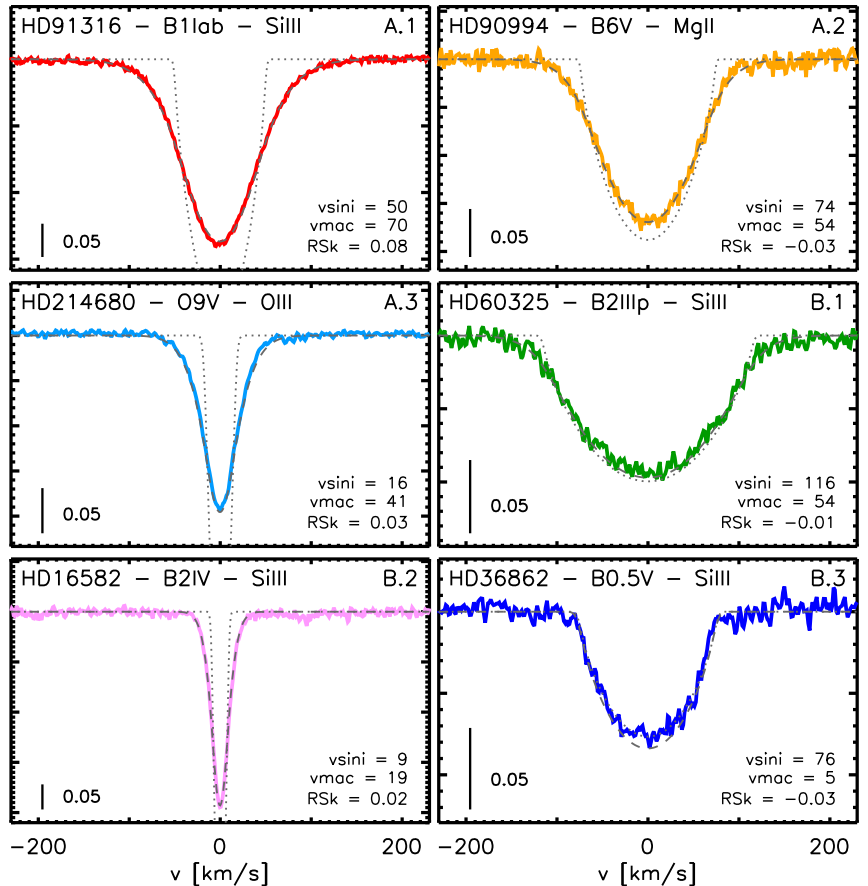

Fig. 2. Illustrative examples of the various types of line profiles found in the IACOB sample of O- and B-type stars. The values of $v \sin i$ and $v_{\text {mac }}$ resulting from the line-broadening and line-asymmetry analyses (see Sect. 3) are quoted in the lower right corners. Each profile was selected to be a representative of the six regions indicated in Fig. 4 (the same color and A)-B) label codes are used here). The profiles are organized from bottom to top and from left to right, following an increasing sequence of $v_{\operatorname{mac}}$ and $v \sin i$, respectively.

As illustrated by Figs. 2 and 3, the width of the profiles ranges from narrow to broad, and its shape varies from clearly roundish, as expected in a rotationally dominated case, to triangular, where the so-called macroturbulent broadening is dominating. Many different combinations of width and shape occur. Most of the profiles are smooth (i.e., do not have any detectable substructure) and symmetric from visual inspection, having similar characteristics as those shown in Fig. 2; however, there is also a non-negligible number of stars showing asymmetric profiles and/or spectroscopic signatures that could be associated with the effect of certain type of pulsations on the line profiles, spots, a magnetosphere, and/or undetected binarity (see some examples in Fig. 3). In particular, regarding pulsating stars, the most clearly detectable cases from single snapshot spectra are those associated with coherent pressure modes as in $\beta$ Cep stars with moderate projected rotational velocities (see, e.g., Panel B.3 in Fig. 3 and Telting et al. 2006, for more illustrative examples) or coherent gravity modes that occur in so-called SPBs (De Cat \& Aerts 2002).

\subsection{Line broadening in $O$ and $B$ stars: $A$ quantitative overview}

Outside the asteroseismology community, it is common to summarize the information about the width and shape of spectral line profiles by means of only two time-independent, line-broadening parameters: $v \sin i$ and $v_{\text {mac }}$. While the first parameter has a well-defined physical meaning, $v_{\text {mac }}$ is only a tuning parameter used to quantify the effect of any other broadening mechanism that is not rotation. For example, as indicated by Aerts et al. (2014), periodic line-profile variability
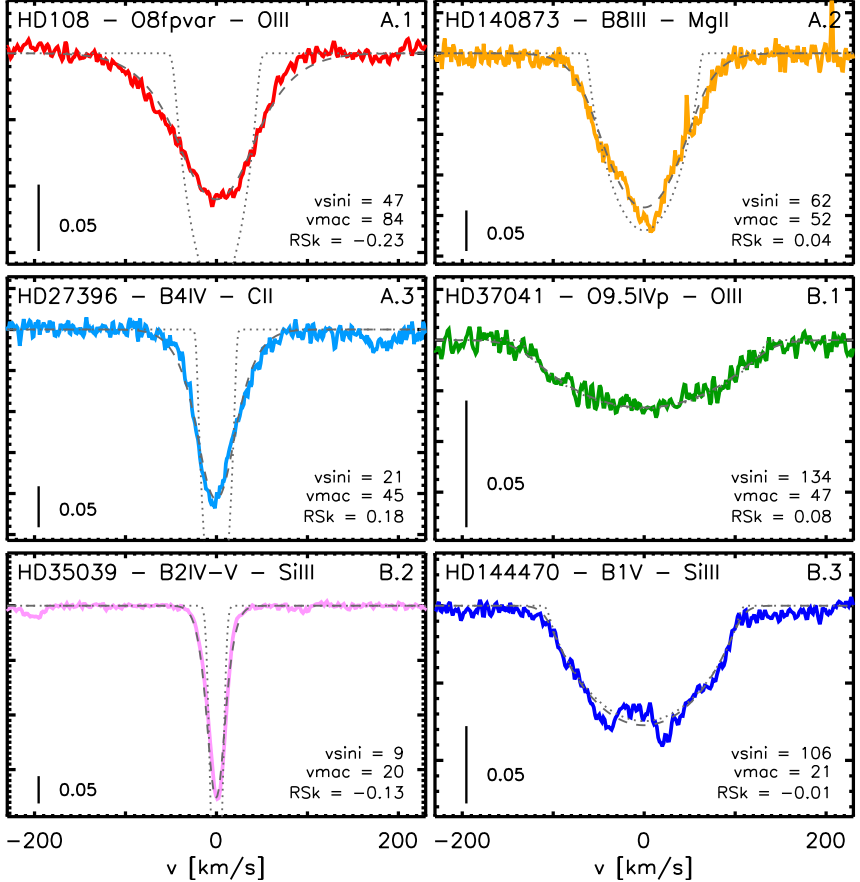

Fig. 3. Same as Fig. 2 but for line profiles that are more asymmetric (A.1, A.3, B.2) or have a more complex structure (A.2, B.3). A case for which only an upper limit of $v_{\text {mac }}$ can be obtained is also illustrated in B.1. In the latter, iacob-broad gives $\left(v \sin i, v_{\text {mac }}\right)=(134,47$; dashed line) as the best fitting solution, but (134, 0; dotted line) is an equally acceptable solution.

caused by surface inhomogeneities or by oscillations in main-sequence B stars can be mimicked by a combination of time-dependent rotational and macroturbulent broadening. Moreover, the value derived for this parameter depends on specific assumptions on the definition of the macroturbulent profile (e.g., radial-tangential versus isotropic Gaussian and the percentage of radial versus tangential components). In addition, as highlighted in SDH14 (see also Sundqvist et al. 2013b; Markova et al. 2014), there is empirical evidence indicating that the methodology described in Sect. 3.1 for disentangling rotation from other sources of line broadening may be failing in some specific cases. This mainly refers to potential limitations, of still unclear origin, in having access to the actual value of the projected rotational velocity in stars with line profiles dominated by the so-called macroturbulent broadening. Therefore, we must handle any quantitative interpretation of the macroturbulent broadening in $\mathrm{O}$ and $\mathrm{B}$ stars in terms of $v_{\mathrm{mac}}$ with care, especially when combining measurements from different sources that might not be using the same techniques or assumptions.

A detailed investigation of the shortcomings of the application of the FT and GOF methodologies in the whole $\mathrm{O}$ and $\mathrm{B}$ star domain, following some guidelines presented in $\mathrm{SDH} 14$, is planed for a subsequent paper of this series (see also some notes in Sect. 5.2.3). In the meantime, we rely on the measurements resulting from the application of the methods described in Sect. 3.1 to provide a complete homogeneous overview of the single snapshot line-broadening characteristics of stars in the whole $\mathrm{O}$ and B star domain. Even though some of the $v \sin i$ and $v_{\text {mac }}$ values quoted in Table 1 may change in the future, the main conclusions presented in these sections will remain since the followed approach provides a representation of the global shape of the line profiles that are valid enough for the purposes of this work. 


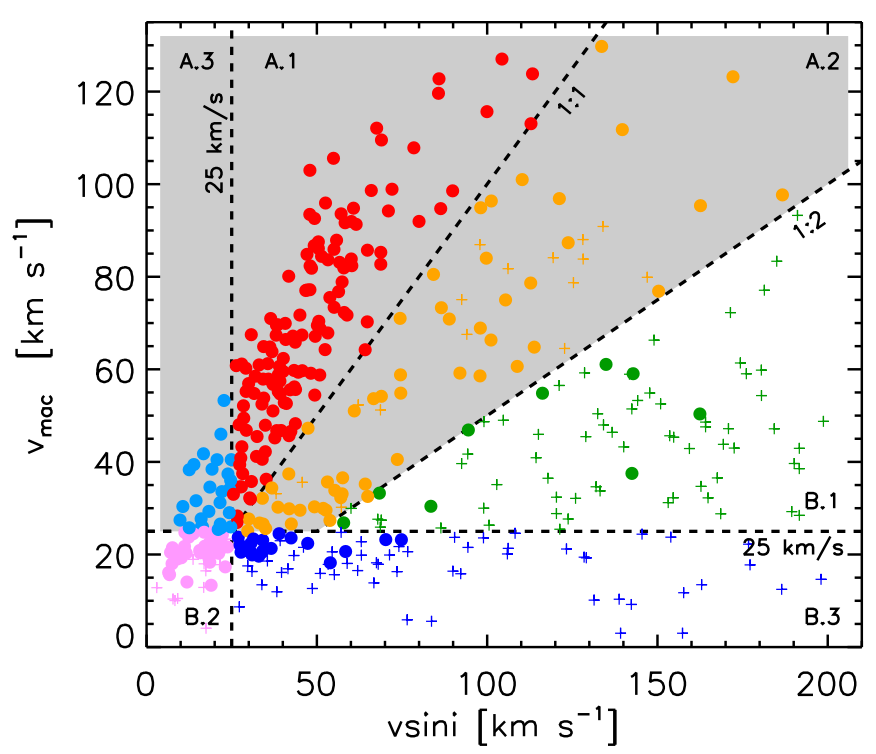

Fig. 4. Line-broadening characterization via $v \sin i$ and $v_{\mathrm{mac}}$ of a sample of 430 Galactic $\mathrm{O}$ and B stars. Crosses correspond to stars for which only an upper limit in $v_{\text {mac }}$ could be determined. Various colors and lines separate stars affected by a different relative rotational and macroturbulent broadening contribution. The $25 \mathrm{~km} \mathrm{~s}^{-1}$ horizontal and vertical lines indicate conservative limitations of the performed methodology (see main text for explanation). Gray-shadowed region groups stars with an important macroturbulent (compared to rotational) broadening contribution.

\subsubsection{Distribution of stars in the $v \sin i-v_{\mathrm{mac}}$ diagram}

The results from the line-broadening analysis of our sample of stars in the $v \sin i-v_{\mathrm{mac}}$ diagram are presented in Fig. 4. As previously found by Lefever et al. (2010), Markova et al. (2014), and by SDH14 using more specific samples in terms of spectral type and luminosity class, the whole diagram is populated below $v_{\text {mac }} \approx 120 \mathrm{~km} \mathrm{~s}^{-1}$, except for a gap found in the low $v \sin i$ regime, where stars with $v_{\text {mac }} / v \sin i \gtrsim 2$ and $v_{\text {mac }} \gtrsim 40 \mathrm{~km} \mathrm{~s}^{-1}$ are absent. This absence may be due to the limitations of the considered methodology (see notes in SDH14). The diagram was divided into six regions depending on the relative contribution of the rotational and macroturbulent broadening and taking into account a conservative lower limit of reliability in the $v \sin i$ and $v_{\text {mac }}$ measurements of $25 \mathrm{~km} \mathrm{~s}^{-1}$ (see SDH14 for more details). Figures 2 and 3 show some illustrative examples of the type of profiles found in each of these regions.

We separate the sample into two broad groups: stars showing an important (or dominant) macroturbulent broadening contribution are indicated in red (A.1), orange (A.2), and cyan (A.3) and grouped by the gray-shadowed region; the other three regions consist of stars in which either rotational broadening dominates (green and dark blue, B.1 and B.3, respectively) or both $v \sin i$ and $v_{\text {mac }}$ are below the limits of reliability of the methodology (pink, B.2). While there are many stars in this latter box for which $v_{\text {mac }}>v \sin i$, the non-rotational broadening could actually be associated with so-called microturbulence (SDH14) or with known sources of pulsational broadening (see Aerts et al. 2014). We hence exclude these stars from those with clearly dominated macroturbulent profiles.

We indicate with crosses those stars for which the iacob-broad analysis was only able to provide upper limits for the value of $v_{\text {mac }}$ (see notes in Sect. 3.1). Panel B.1 in Fig. 3 shows one of these cases. Most of the stars are concentrated in the region where $v_{\mathrm{mac}} / v \sin i \leq 0.5$. This is expected since the extra broadening only produces a small effect on the wings of the line profile when rotational broadening dominates. Although they are not shown in Fig. 4, we found that this situation extends to all stars in the IACOB sample with $v \sin i>200 \mathrm{~km} \mathrm{~s}^{-1}$. Therefore, we decided to exclude these stars from the discussion below after checking that this decision does not modify any of the conclusions presented in this paper.

Despite the multi-epoch character of the IACOB spectroscopic dataset, there may still be some cases of undetected binarity or composite spectra. We have only one spectrum for some of the stars in the database and some composite spectra may not be detected with the (minimum 3) spectra we have for most of the targets. Some stars are already indicated as suspicious in view of the shape of the line profiles (e.g., HD 140873 in Fig. 3); however, some others can still remain unidentified, especially when we only have one spectrum. We hence must keep in mind that there may be some cases in which the single snapshot line-broadening analysis presented here could end up in spurious results. In particular, a composite spectrum with two lines that are not clearly separated can be artificially fitted with a line profile having a larger $v \sin i$ and, especially, a larger $v_{\text {mac }}$ than any of its individual components.

\subsubsection{Macroturbulent broadening across the upper part of the sHR diagram}

The location of the stars from groups A and B in the sHR diagram is presented separately in the left and middle panels, respectively, of Fig. 5. We use the same colors and symbols as in previous figures. The non-rotating evolutionary tracks for solar metallicity from Ekström et al. (2012) are also shown for guidance purposes. The typical uncertainties in $\log T_{\text {eff }}$ and $\log \mathscr{L}$ (see Sect. 3.3) are indicated with a black cross in the bottom left corner of each panel. These two panels are complemented with a third one that shows the same distribution of stars but from a different perspective. This time, we plot all stars together (except those for which $v_{\text {mac }}$ could not be properly determined, i.e., the cross symbols in Figs. 4 and 5), using the same color code, but scaling the size of the symbols with $v_{\text {mac }}$. All together, these figures present, for the first time, a homogeneous and statistically significant overview of the (single snapshot) line-broadening properties of stars in the whole $\mathrm{O}$ and B star domain.

We highlight below the main results that can be extracted from an inspection of Fig. 5, regarding the distribution of stars in the sHR diagram in terms of line-profile shape characteristics.

The upper part of the sHR diagram, including the $\mathrm{O}$ stars and B Sgs or, roughly speaking, stars with initial masses $\gtrsim 15 M_{\odot}$, is basically populated by stars with a remarkable non-rotational broadening component (red, orange, and cyan circles). In this region of the diagram, there are only a few stars from group $\mathrm{B}$ (mostly green crosses with $v \sin i>130 \mathrm{~km} \mathrm{~s}^{-1}$ ).

The relative number of stars with profiles dominated by macroturbulent broadening (red circles) becomes much smaller when moving down in the sHR diagram. There are a few outliers in the lower part of the diagram with high values of $v_{\text {mac }}$; however, these may actually be non-detected spectroscopic binaries (see note in Sect. 4.2.1). Indeed, new observations obtained during the development of this paper have allowed us to confirm this hypothesis in a couple of targets (now excluded from the final sample). Other similar cases may still remain in the sample. 


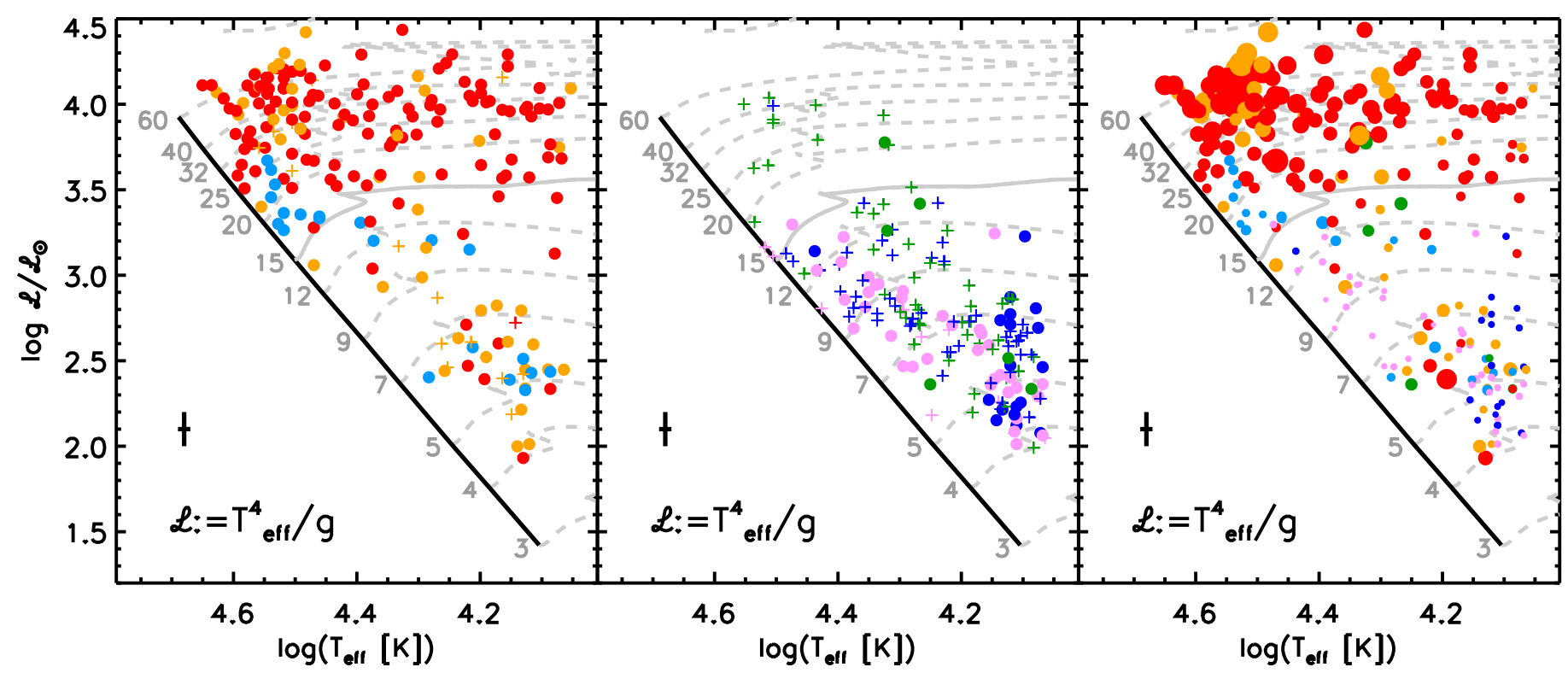

Fig. 5. Location of the stars from groups A (left panel) and B (middle panel), as defined in Sect. 4.2.1, in the sHR diagram. Same color code as in Fig. 4. The Geneva, non-rotating evolutionary tracks (Ekström et al. 2012) are overplotted for guidance purposes. The black cross to the left shows the typical uncertainties in $\log T_{\text {eff }}$ and $\log \mathscr{L}$ (assuming conservative uncertainties in $T_{\text {eff }}$ and $\log g$ of $5 \%$ and 0.15 dex, respectively, and no correlation between both quantities). (Right panel) All stars from groups A and B (except those for which $v_{\text {mac }}$ could not be properly determined) joined together with symbol sizes proportional to $v_{\text {mac }}$.

Contrarily to the homogeneity, in terms of line-broadening properties, found in $\mathrm{O}$ stars and $\mathrm{B} \mathrm{Sgs}$, the situation is much more heterogeneous in the $\mathrm{B}$ dwarf and $\mathrm{B}$ giant region $\left(\lesssim 15 M_{\odot}\right)$. The only common characteristic in this lower part of the sHR diagram is that the amount of non-rotational broadening (quantified as $v_{\mathrm{mac}}$ ) is much smaller in absolute terms in comparison with stars with larger masses (see right panel in Fig. 5). Apart from this, below $\log \mathscr{L} / \mathscr{L}_{\odot} \sim 3.5$, stars with different types of profiles are not concentrated in specific regions of the diagram.

We interpret these results as empirical evidence of the existence of various types of non-rotational broadening agents acting in the realm of massive stars. Even though all of these stars can be quantitatively characterized in terms of $v_{\mathrm{mac}}$, and quoted as macroturbulent broadening, from a practical point of view their physical origin can be different. Indeed, it is natural to think that some of them could be acting at the same time, with a different relative contribution to the line profiles, depending on the specific properties of the star at a given moment during its evolution.

Under this scenario, the distribution of stars shown in Fig. 5 indicates that, below $\approx 15 M_{\odot}$ (or, equivalently $\log \mathscr{L} / \mathscr{L}_{\odot} \sim 3.5$ ) the final shape of the stellar lines depends on a combination of different factors that are not only controlled by $T_{\text {eff }}, \log g$, and/or $v \sin i$. This statement is in agreement with Aerts et al. (2014, see also notes in Sect. 5.2.3), who showed that, in the B main-sequence star domain, the presence of stellar spots, surface inhomogeneities, and stellar oscillations are effective sources of (variable) line broadening. Therefore, in this case, we should avoid using the term macroturbulent broadening, or at least, be aware that the sources of broadening in these stars may have nothing to do with what we call macroturbulent broadening in other regions of the HR diagram (e.g., the Sun, cool stars, A-type stars, or even O stars and B Sgs). As indicated by Aerts et al. (2014) the correct identification of the broadening agents should not rely on a single snapshot, but on multi-epoch observations.
Above $\approx 15 M_{\odot}$ we find a more homogeneous distribution of profile types, suggesting one main broadening agent in the upper part of the sHR diagram, whose relative contribution becomes gradually less important for lower masses. Since very similar profiles are found for stars covering a broad range of effective temperatures, gravities, rotational velocities, and wind properties, none of these stellar properties seems to be a key factor in the occurrence of this extra broadening. In addition, the fact that this broadening agent is operating in a very similar way for stars in different evolutionary stages (from the $\mathrm{O}$ dwarf to the late-B supergiant phases) can be considered a strong empirical constraint to any scenario trying to explain its physical origin.

Figure 6 reinforces these statements and also adds, in a more quantitative way, interesting information about the dependence of $v_{\mathrm{mac}}$ on $\log \mathscr{L}$ and $T_{\text {eff }}$. Again, both panels of Fig. 6 indicate the absence of a remarkable connection between $v_{\text {mac }}$ and any of these two quantities for stars with $\log \mathscr{L} / \mathscr{L}_{\odot} \lesssim 3.5$ (highlighted using black open circles). Above this value, where we postulate that only one main broadening agent (in addition to rotation) begins to dominate over the rest, a clear positive correlation between $v_{\text {mac }}$ and $\log \mathscr{L}$ is detected. Since, the quantity $\mathscr{L}$ is proportional to $L / M$ or, equivalently, to $\Gamma_{\text {Edd }}$ or $g_{\text {rad }} / g_{\text {grav }}$ (see Langer \& Kudritzki 2014), one could argue that $v_{\text {mac }}$ becomes larger when the impact of radiation pressure (at least in the outer, but still subsonic envelope) increases. As we discuss in Sect. 5.2.1, however, other effects may also explain this correlation (see also Grassitelli et al. 2015a).

Circles not surrounded in black in the bottom panel of Fig. 6 show the dependence of $v_{\text {mac }}$ on $T_{\text {eff }}$ for the case of $\mathrm{O}$ stars and B Sgs (cf. stars with $\log \mathscr{L} / \mathscr{L}_{\odot}>3.5$ in Fig. 5). In addition to the decrease of $v_{\mathrm{mac}}$ from the early- to the late-B supergiants already pointed out by previous studies (see, e.g., Dufton et al. 2006; Lefever et al. 2007; Markova \& Puls 2008; Fraser et al. 2010; Markova et al. 2014; Simón-Díaz \& Herrero 2014), the figure seems to indicate that there is also a small decrease of this quantity from the late-O and early-B $\operatorname{Sgs}\left(\log T_{\text {eff }} \sim 4.5\right)$ toward 

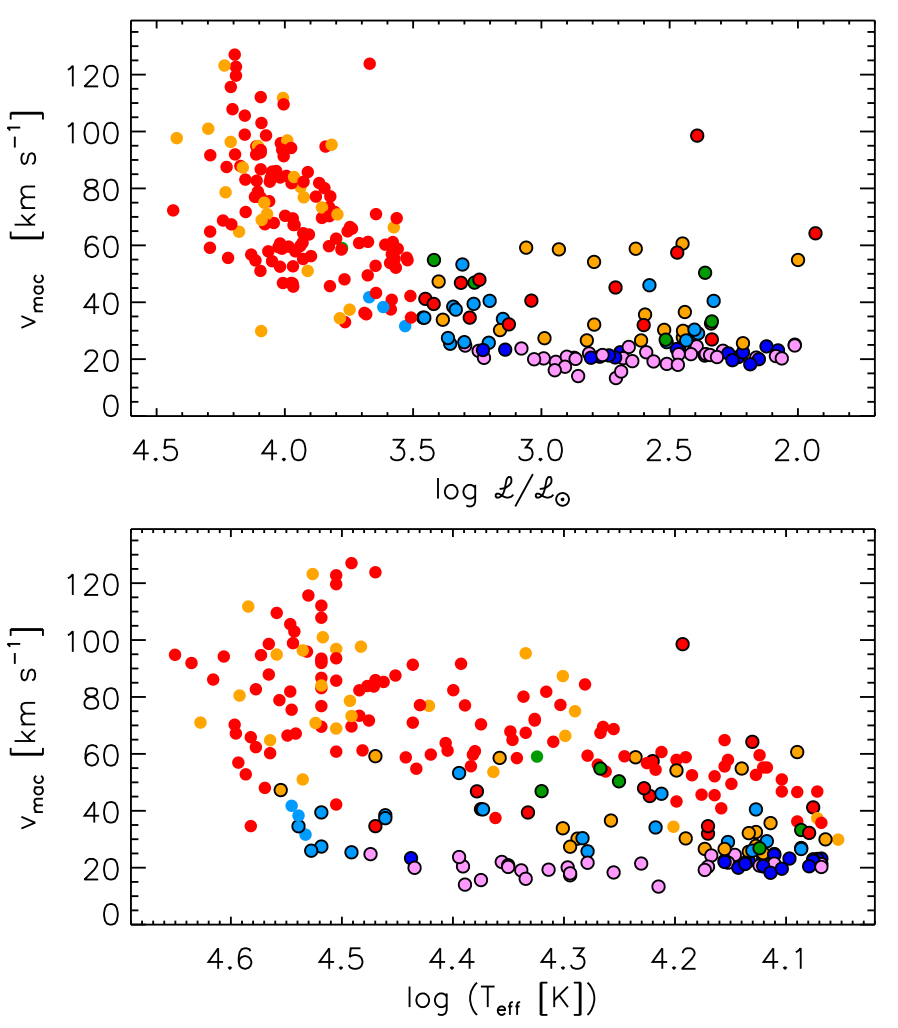

Fig. 6. Dependence of $v_{\text {mac }}$ on $\log \mathscr{L}$ and $T_{\text {eff }}$ for the same stars included in the right panel of Fig. 5. Stars with $\log \mathscr{L} / \mathscr{L}_{\odot}<3.5$ are shown in both panels with open black circles for better identification.

the hotter $\mathrm{O}$ giants and dwarfs $\left(\log T_{\mathrm{eff}} \gtrsim 4.5\right)$. Last, late-O dwarfs are characterized by having the lowest values of $v_{\text {mac }}$ among the luminous OB stars (see cyan circles with $\log T_{\text {eff }} \gtrsim 4.45$ and $\log \mathscr{L} / \mathscr{L}_{\odot}>3.2$ in Figs. 5 and 6).

\section{Discussion}

\subsection{On the pulsational origin of macroturbulent broadening in $O B$ stars}

Howarth (2004) presented a summary of different scenarios that had been proposed by that time to explain the existence of what we traditionally call macroturbulent broadening in $\mathrm{O}$ stars and B Sgs. In brief, these scenarios invoke the effect of stellar winds, pulsations, differential rotation, turbulent motions, and rotationally induced tangential turbulence on the stellar lines. In all cases, there is an implicit assumption that the occurrence of this spectroscopic feature is associated with large-scale velocity fields globally affecting the line-formation region.

From all these scenarios, macroturbulent broadening in the OB star domain being a spectroscopic signature of stellar oscillations is presently the most strongly considered hypothesis. Although this possibility has not been yet definitely proven from an observational point of view, works by Aerts et al. (2009, 2014), Simón-Díaz et al. (2010), Simón-Díaz (2015), Aerts \& Rogers (2015), and Grassitelli et al. (2015a) have produced encouraging results in this direction.

Aerts et al. (2009) revived the pulsational scenario, already proposed a long time ago by Lucy (1976), showing that the shape of the metal line profiles observed in the high-resolution spectra of B Sgs can naturally be reproduced by the combined velocity broadening effect of hundreds of low-amplitude, non-radial gravity-mode pulsations. This work served to support theoretically, for the first time, stellar oscillations as a viable explanation for macroturbulent broadening in OB stars.

Simón-Díaz et al. (2010) provided, for the first time, firm observational evidence for a strong correlation between the amount of non-rotational broadening affecting the line profiles in a sample of $13 \mathrm{OB}$ Sgs and the peak-to-peak amplitude of variation of the third moment (skewness) of the lines. Interestingly, very similar trends were obtained one year before from the simulations by Aerts et al. (2009). This encouraging result helped to further support, this time from the observational point of view, the hypothesis that stellar oscillations are the most probable physical origin of macroturbulent broadening in B Sgs.

In this context, we remark that Aerts et al. did not consider the excitation of the modes but rather studied the impact of a certain type of oscillations on the line-broadening and line-profile variations. In particular, Aerts et al. (2009) highlighted the importance of considering a large range of mode degrees (i.e., leading to a dense frequency spectrum of modes as, for example, occurs in gravity-mode pulsators) to reproduce, thanks to their collective effect, the observed characteristics of the macroturbulent profiles in terms of global shape and structure.

Heat-driven non-radial coherent pressure $(p)$ or gravity ( $g$ ) modes excited by the $\kappa$ mechanism in the iron opacity bump are clear candidates to produce stellar oscillations with these characteristics in certain regions of the HR diagram. However, these are not the only possibility. Stochastically excited oscillations giving rise to a whole spectrum of waves driven by turbulent pressure fluctuations initiated in inefficient (in transporting energy) subsurface convection zones (Grassitelli et al. 2015a,b) or by the interface of the convective core and the radiative envelope (Rogers et al. 2013; Aerts \& Rogers 2015) are other options that have been proposed. All these causes (in addition to possibly others not mentioned here, for example, strange modes, especially in the high $L / M$ regime) could be acting together, contributing with a different weight depending on the specific star.

\subsection{Using observations to evaluate the pulsational scenario of macroturbulent broadening in OB stars}

There are many aspects that must be considered to evaluate the suggested macroturbulent-pulsational broadening connection and, in the framework of this scenario, identify the excitation mechanism starting the instabilities which, ultimately lead to the extra broadening of the line profiles. In this context, it is important to remark that different types of oscillations, combined with other geometrical factors, can give rise to different spectroscopically observable features (mainly in the form of variable line-profile shape, asymmetry, and broadening). The works by Schrijvers et al. (1997) and Telting \& Schrijvers (1997) give a comprehensive illustration of predicted line-profile variations originated by adiabatic non-radial pulsations in rotating stars. Therefore, we ideally have to assess, for the various proposed driving mechanisms, how the predictions about all these (time-dependent) line-profile characteristics confront with the observations. In this regard, we basically enter into the domain of asteroseismology.

Time-resolved spectroscopy is the most suitable way to unravel whether the line-profile behavior is connected with strict periodicity as occurs for long-term coherent modes or rather with stochastic excitation. It is also a very powerful tool to evaluate whether the line broadening and variability is connected with a few excited modes or is the result of collective effect of waves. 

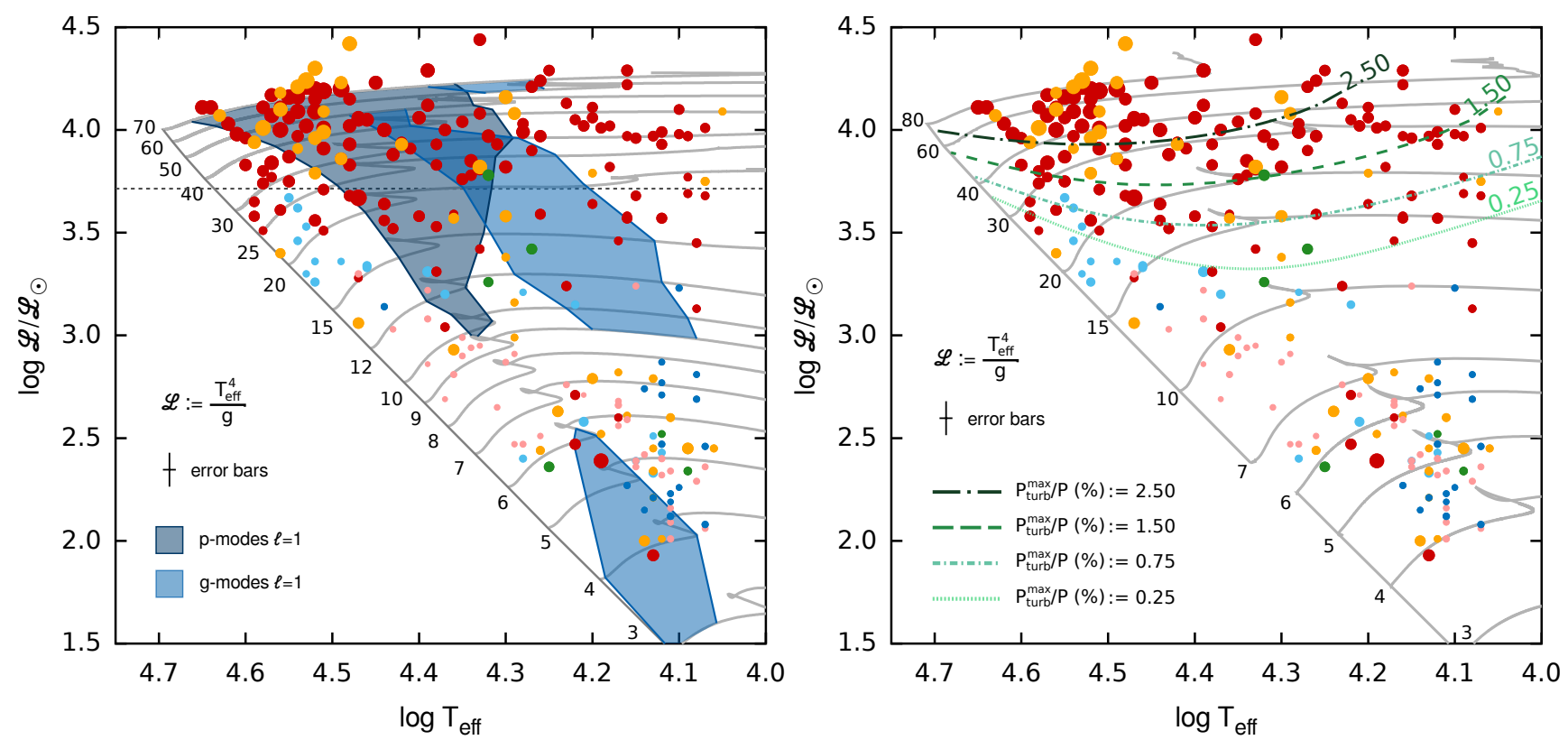

Fig. 7. Model predictions for (left) heat-driven $p$ and $g$ modes with $\ell=1$ computed by Godart et al. (2016), and (right) the maximum fraction of turbulent pressure over total pressure as derived by Grassitelli et al. (2015a). Same observations as those included in right panel in Fig. 5, with the color code described in Sect. 4.1 and symbol sizes proportional to $v_{\text {mac }}$. Each panel considers different evolutionary tracks for coherence with the models considered in the original papers performing the computations.

Last, it can also help to identify whether there is a dominance of $p$ or $g$ modes acting on the line hprofiles. However, following Simón-Díaz et al. (2012), we also illustrate in next section how the empirical distribution of the single snapshot line-broadening properties in the sHR diagram discussed in Sect. 4.2.2 can be used as a complementary (and definitely less expensive compared to time-resolved spectroscopy) tool to assess the viability of the possible connection between macroturbulent broadening in OB stars and some of the excitation mechanisms of (possibly-)cyclic surface motions indicated above.

\subsubsection{Distribution of macroturbulent broadening in the sHRD and driving mechanisms of stellar oscillations}

In Simón-Díaz (2015) we used part of the observational sample presented here to investigate possible correlations between the location of stars with a remarkable macroturbulent broadening contribution and the high-order $g$-mode instability strips from Miglio et al. (2007a) and Godart (2011). Later on, the same observations were included in the work by Grassitelli et al. (2015a) to assess the proposed connection between turbulent pressure fluctuations initiated in inefficient (in transporting energy) subsurface convection zones and the occurrence of macroturbulent broadening in OB stars.

In Fig. 7 we present again the comparison of observations and model predictions for both scenarios but, this time, using the complete, cleaned sample discussed in Sect. 4.2.2. Similar to right panel in Fig. 5, we combine all stars from groups A and B in the same plot, using the color code defined in Fig. 4, symbol sizes proportional to $v_{\mathrm{mac}}$, and excluding those stars for which this quantity could not be properly determined (see Sect. 4.2.1). For comparative purposes with the observational dataset used in Simón-Díaz (2015) and Grassitelli et al. (2015a), the sample considered here includes $\approx 150$ stars more and excludes $\approx 20$ stars subsequently detected to be spectroscopic binaries using new multi-epoch observations compiled since then.
The left panel of Fig. 7 is an updated version of Fig. 2 from Simón-Díaz (2015) in which we present results in the sHR diagram (instead of the $\log g-\log T_{\text {eff }}$ diagram), and include the instability strips for heat-driven $p$ and $g$ modes with $\ell=$ 1 resulting from a new homogeneous set of adiabatic and non-adiabatic computations by Godart et al. (2016) in the whole 3-70 $M_{\odot}$ range. As already commented in Simón-Díaz (2015), the presence of large red circles in the upper part of the sHR diagram outside the predicted instability domains implies a strong empirical challenge to non-radial modes excited by a heat mechanism being the main physical driver of the non-rotational broadening affecting $\mathrm{O}$ stars and B Sgs (except maybe for the early-B Sgs, located inside the post-TAMS $g$-mode strip for stars with $M \gtrsim 10 M_{\odot}$; see also notes in Aerts et al. 2009). Indeed, even only considering the stars located inside instability domains we would expect a different effect on the profile shape of the low degree $p$ and $g$ modes, where the latter are characterized by having a denser frequency spectrum of excited modes. But this is not what the observations are telling us (see Sect. 4.2.2 and 5.2.3).

However, this is not the last word concerning this scenario. As indicated above, the instability domains presented in the left panel of Fig. 7 correspond to computations for low degree modes. The situation may improve when accounting for the predictions for higher degree modes (indeed, the simulations by Aerts et al. 2009, considered 241 excited modes with degree $\ell$ from 1 to 10). In addition, the use of different input parameters (e.g., opacities, metal mixture, metallicity, and overshooting parameter) or even evolutionary codes may alter the results (see, e.g., Miglio et al. 2007a,b; Zdravkov \& Pamyatnykh 2008; Salmon et al. 2012; Turck-Chièze \& Gilles 2013; Martins \& Palacios 2013; Castro et al. 2014, for recent illustrations of these effects). For reference, the instability domains presented in left panel of Fig. 7 correspond to computations performed with the ATON evolutionary code (Ventura et al. 2008) assuming the OPAL opacity tables (Iglesias \& Rogers 1996), a metallicity $Z=0.015$, and the 
metal mixture from Grevesse \& Noels (1993). See Godart et al. (2016) for further details.

It will hence be interesting to investigate how all these effects may help to bring instability domains, in particular, those connected with $g$ modes, toward the ZAMS in the Oand early-B star domain, and toward cooler temperatures in the $\mathrm{B}$ supergiant region, in a reasonable way. In this context, we already indicate, for example, that the instability strips recently computed by Moravveji (2016) based on the new Fe opacity measurements by Bailey et al. (2015) reach all the way to the ZAMS. Concerning the late-B Sgs oscillations excited by the $\epsilon$ mechanism (Noels \& Scuflaire 1986; Unno et al. 1989; Moravveji et al. 2012b) may play an important role. Last, the predicted occurrence of adiabatic strange modes for $\log \mathscr{L} / \mathscr{L}_{\odot}>3.7$ are not to be forgotten; these refer to modes for which the excitation in the iron opacity bump because the $\kappa$ mechanism is enhanced by the large amplitude of the mode trapped into a superficial cavity (see, e.g, Saio et al. 1998, and references therein; Aerts et al. 2010a). Some of these points are explored and discussed in more detail in the parallel work by Godart et al. (2016).

The right panel of Fig. 7 is an adaptation of Fig. 4 from Grassitelli et al. (2015a) in which we overplot the isocontours of the quantity $P_{\text {turb }}(\max ) / P$ (maximum fraction of turbulent pressure over total pressure) as predicted by Grassitelli et al. (2015a) to the distribution of line-broadening properties of the updated sample of stars in the sHR diagram. For completeness, the information presented in this panel must be complemented with Fig. 5 in Grassitelli et al. (2015a) in which $v_{\text {mac }}$ is plotted against $P_{\text {turb }}(\max ) / P$.

As already pointed out by Grassitelli et al., the good correlation found between these two quantities renders the turbulent pressure scenario very promising, at least regarding the upper part of the HR diagram. Indeed, the predicted behavior of the strength of this driving mechanism with $T_{\text {eff }}$ and $\mathscr{L}$ may explain the similarity of line profiles found in the whole $\mathrm{O}$ and B Sgs domain, this scenario implies only one broadening agent behaving in a similar way in the whole range, and the strong dependence of $v_{\text {mac }}$ on $\mathscr{L}$ established empirically (see Sects. 4.2.2). However, there is still a very important point to be evaluated in this scenario. Grassitelli et al. only provided indirect arguments indicating that turbulent pressure fluctuations initiated in subsurface convection zones may drive high-degree oscillations. One could extend further this argument, indicating that we might be dealing with stochastically excited waves behaving in a similar way to the (gravity) modes assumed in the simulations by Aerts et al. (2009). However, this entire line of argument must still be directly proven, especially regarding the fact that we must end up reproducing the observed amount of line broadening and profile shapes characterized by not having any detectable substructure in the spectral lines. The latter is indeed an important observational constraint to be taken into account when assessing any scenario to explain macroturbulent broadening in OB stars.

Some work along this line has recently been performed by Aerts \& Rogers (2015), who also proposed a third (maybe complementary) scenario in which macroturbulent broadening is related to the occurrence of convectively driven internal gravity waves (IGWs). Aerts \& Rogers (2015) based their study in two-dimensional (2D) numerical simulations as in Rogers et al. (2013) and showed that the stochastic spectrum of running waves caused by IGWs may lead to detectable line-profile variability and explain the extra broadening observed in the line profiles of OB stars. However, given the intense CPU time required by the computations they could only perform the investigation for a $3 M_{\odot}$ main-sequence star. Therefore, we still can not compare the predictions of this scenario with the empirical distribution of line-broadening properties in the sHR diagram as in previous cases.

New simulations of such internal gravity waves across stellar evolution is hence a very interesting line of future work in this respect. In addition, as indicated in Sect. 5.2, and illustrated in next section, the compilation and analysis of spectroscopic time series will also provide interesting clues to identify the type of waves and the associated driving mechanism producing the different sources of non-rotational broadening and line-profile variability detected in $\mathrm{O}$ stars and $\mathrm{B} \mathrm{Sgs}$ (as asteroseismology has efficiently shown in the case of B stars, among many other type of stars across the HR diagram).

\subsubsection{Macroturbulent broadening and line-profile asymmetry}

Apart from broadening effects, line asymmetries and shifts are important observables for the characterization of the seismic properties of stars, in particular if we include a description of how these line properties vary with time (cf. Chaps. 5 and 6 of Aerts et al. 2010b). It is hence of interest to incorporate quantities accounting for this information to achieve any physical interpretation of macroturbulent broadening, especially in the context of stellar oscillations.

The velocity moments of the line profile (Balona 1986; Aerts et al. 1992; Schrijvers et al. 1997) have been proven to be powerful tools to characterize the temporal behavior of line asymmetry independent of the physical cause that is originating it. An interesting work in this respect is Schrijvers et al. (1997), in which line-profile asymmetry due to a long lifetime oscillation mode of degree $\ell$ and azimuthal order $m$ in rotating stars is shown for a large variety of oscillation and rotational parameters; the latter was quantified in terms of the rotational frequency $\Omega$ and the inclination angle $i$ of the star. The predicted moment variations along with the line profiles are presented for many combinations of $(\ell, m, i, \Omega)$, showing that asymmetry is obvious from the moments even though the profiles themselves are seemingly symmetric. Along the same lines, Briquet et al. (2004) demonstrated that the moments of a line profile allow us to distinguish line asymmetry due to surface spots versus oscillations and Hekker \& Aerts (2010) showed the utility of using the moments even in the case of stochastically excited oscillations. While these are just three illustrative examples, many other works in recent decades have shown interest in quantifying line-profile variations by means of the line moments.

In this section, we use our single-epoch observational dataset to investigate possible correlations between $v_{\text {mac }}$ and the amount of asymmetry of the line profiles. Being aware of the limitations of this study, given the expected variability of the considered quantities with time (see, e.g., Simón-Díaz et al. 2010, Aerts et al. 2014), we still consider of interest the discussion of the results of such a single snapshot approach while awaiting for the complete ongoing time-resolved IACOB observations. To this aim we plot in Fig. 8 the dimensionless ratio $v_{\text {mac }} / v \sin i$ versus the, also dimensionless, quantity $R S k$ (relative skewness) defined in Sect. 3.2. For a cleaner presentation of the results, we divide the figure into three panels. From top to bottom we include (a) the whole sample of 430 stars discussed in Sect. 4.2 without uncertainties in $R S k$ over plotted; (b) only those stars with clear asymmetric profiles; and (c) the remaining stars, in which the measured $R S k \pm \sigma_{\mathrm{RSk}}$ is compatible with a symmetric profile. 
S. Simón-Díaz et al.: New observational clues to understand macroturbulent broadening in massive O- and B-type stars

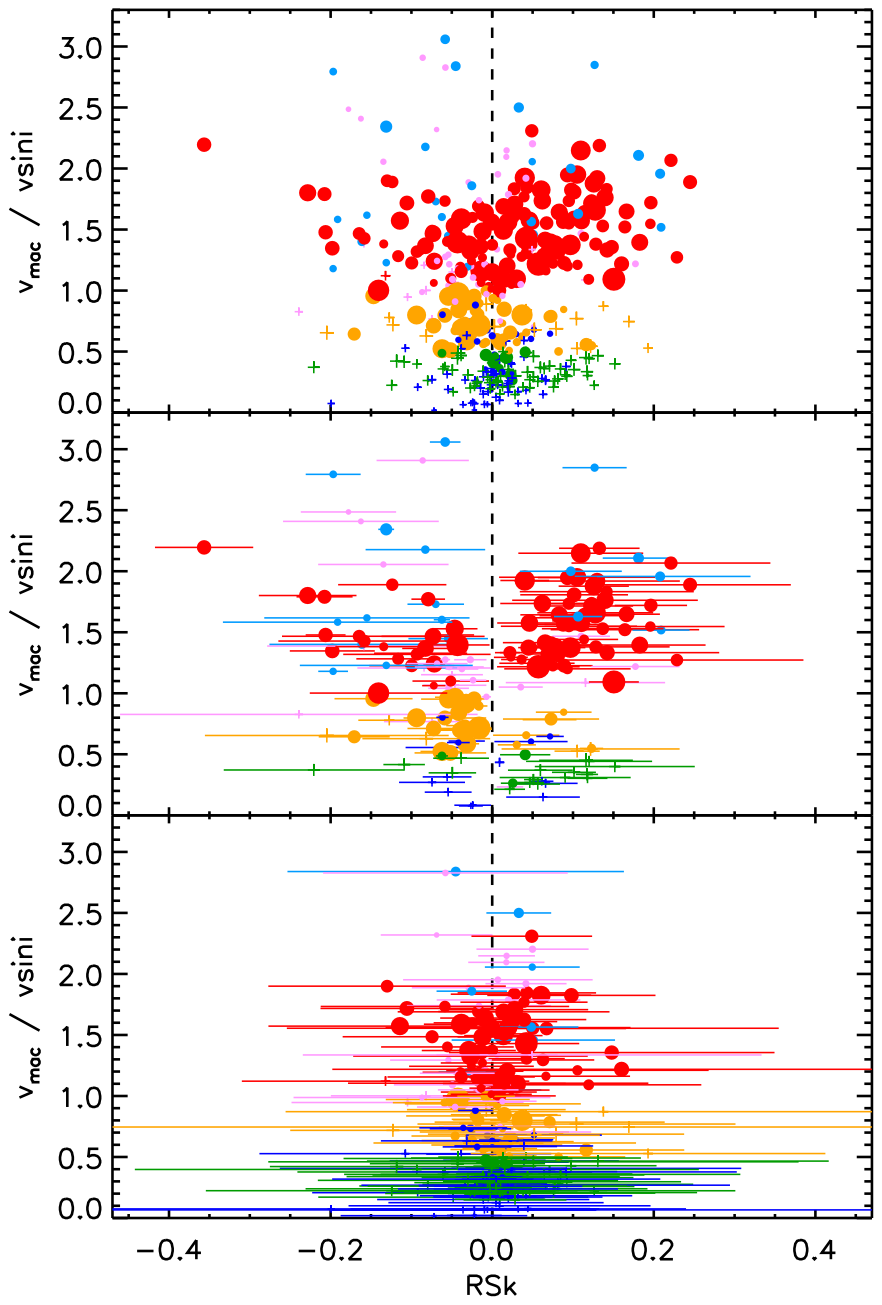

Fig. 8. Macroturbulent broadening (normalized to $v \sin i$ ) vs. asymmetry for (top) all 430 stars considered in this paper with no uncertainties overplotted; (middle) only those stars with clear asymmetric profiles; (bottom) stars with line profiles in which the measured value of the quantity $R S k$ is compatible with a symmetric profile given the associated uncertainty. Same colors and symbols as in Figs. 4 and 5. The size of the symbols scales with $v_{\text {mac }}$.

Figure 8 is complemented with the information provided in Table 2, where we indicate, for each of the subgroups defined in Fig. 2, the percentage of stars that have line profiles with clear positive or negative relative skewness or that are compatible with $R S k=0$.

The first interesting result to be highlighted is the presence of a remarkable number of stars with clearly identified asymmetric profiles (almost half of the global sample, see last row in Table 2). Among these stars, the percentage of profiles with positive or negative skewness is basically the same $(\approx 22 \%)$. This result can be interpreted as empirical evidence of line asymmetries that are associated with a variable phenomenon that is producing a time-dependent distribution of motions in the line formation region with an average velocity close to zero. Since we are considering snapshot measurements for a large number of stars, and each of these measurements can be randomly picked at any instance during the variation cycle, such a phenomenon would result in a distribution of points similar to that shown in Fig. 8.

This would exclude, for example, any wind-type variability or differential rotation as the main cause of the asymmetries, at
Table 2. Summary of results presented in Fig. 8 regarding the quantity $R S k$.

\begin{tabular}{lcccccc}
\hline \hline & & & \multicolumn{2}{c}{ Asymm. } & & Symm. \\
\cline { 4 - 5 } \cline { 5 - 5 } Color & Label & $\#$ & $R S k<0$ & $R S k>0$ & \\
\hline Red & A.1 & 137 & $17 \%$ & $41 \%$ & \\
Orange & A.2 & 70 & $33 \%$ & $12 \%$ & & $52 \%$ \\
Cyan & A.3 & 27 & $48 \%$ & $22 \%$ & \\
Green & B.1 & 73 & $7 \%$ & $19 \%$ & & $30 \%$ \\
Pink & B.2 & 49 & $39 \%$ & $10 \%$ & & $51 \%$ \\
Blue & B.3 & 76 & $12 \%$ & $8 \%$ & \\
Total & - & 430 & $21 \%$ & $22 \%$ & & $50 \%$ \\
\hline
\end{tabular}

Notes. Asymmetric (symmetric) profiles refer to cases with $|R S k|-$ $\sigma_{\text {RSk }}$ that are greater (less) than zero, respectively. Stars separated by subgroups as defined in Sect. 4.2.1 (see also Fig. 2). For reference, formally, a perfectly symmetric line implies $R S k=0$, while positive (negative) values of the quantity $R S k$ represent a redward (blueward) asymmetric line (see, e.g., panels A.3 and A.1 in Fig. 3, respectively).

least generally speaking, as these two cases imply motions that are going along with an average velocity that is different from zero (i.e., it is very difficult to obtain line asymmetries that swap signs). In contrast, stellar oscillations fulfill this requirement.

Table 2 also shows that, except for the cases in which the relative contribution of the macroturbulent broadening is negligible (i.e., B. 1 and B.3, where $v_{\operatorname{mac}} / v \sin i<0.5$ ), the percentage of stars with clear asymmetric profiles is always larger than 45\%. In addition, back in Fig. 8, the larger concentration of profiles found among the most asymmetric lines are those with a dominant contribution of the macroturbulent broadening (red dots). All this suggests the potential connection between macroturbulent broadening and line asymmetries and, hence, following the argument above, a variable phenomenon.

As indicated by Aerts et al. (2009, 2014) and Simón-Díaz et al. (2010), the association between stellar oscillations, the detected asymmetries and the so-called macroturbulent broadening is a promising possibility. This scenario seems to be also supported, at least in a global sense, by the distribution of stars in the $v_{\mathrm{mac}} / v \sin i$ vs. $R S k$ diagram presented in Fig. 8. However, a closer inspection of the distribution of points in each of the subgroups considered in Table 2 indicates that the situation may be more complex than just assuming only one type of physical driving of the (likely variable) line broadening and line asymmetry in the whole O- and B-type star domain. For example, the fact that the $R S k$ distribution in the middle panel of Fig. 8 is weighted to positive values in group A.1 (red) and negative values in groups A.2 (orange), A.3 (cyan), and B.2 (pink; see also Table 2) deserves further attention in future studies incorporating time-resolved information about all the considered quantities.

One interesting possibility to be investigated would be related to the different effect that pressure and gravity-dominated oscillations (which have a dominant radial and transversal component, respectively) may have on snapshot properties of the line profiles in a large sample of stars, since their amplitude distribution across the oscillation cycle in terms of asymmetry is different (e.g., Chap. 6 in Aerts et al. 2010b). Other options to be evaluated would be the effect that stellar winds and magnetic fields may have on the skewness properties of the considered diagnostic lines.

The analysis of time-series observations of a large sample of stars covering the various types of line profiles allows us 
to investigate whether the observed distribution of points in Fig. 8 is connected to the type of oscillations indicated above. In addition, the incorporation of information about the stellar wind and magnetic properties of the considered stars, along with the single or composite status of the analyzed spectra, serves to identify those cases in which the shape and variability of the considered diagnostic lines can be affected by other effects apart from stellar oscillations.

\subsubsection{Macroturbulent broadening and line-profile variability: OB stars vs. well known pulsating and spotted B stars}

With the aim of supporting the pulsational hypothesis to explain macroturbulent broadening in massive $\mathrm{O}$ and $\mathrm{B}$ stars and validating current methodologies to estimate projected rotational velocities in these stellar objects, Aerts et al. (2014) investigated a sample of well-known spotted and pulsating B stars with time-dependent profiles. They analyzed available multi-epoch spectroscopic observations by means of similar techniques as those described in Simón-Díaz \& Herrero (2014) and used in this paper. They found that both line-broadening parameters vary (in anti-phase with each other) appreciably during the pulsation cycle whenever the pulsational and rotational velocity fields have similar magnitudes. In addition, the macroturbulent velocities derived while ignoring the pulsations can vary by tens of $\mathrm{km} \mathrm{s}^{-1}$ during the pulsation cycle. This study hence warned about misinterpretations based on a $v \sin i+v_{\text {mac }}$ single snapshot approach for the line-broadening characterization of stars with an important non-rotational broadening contribution to the line profiles.

Here we use already available multi-epoch observations to tackle the question of how this situation compares with the case of OB stars with line profiles dominated by macroturbulent broadening and provide some first results using time-resolved spectroscopy to assess the pulsational hypothesis in these more massive objects without awaiting the full time-series data.

We selected for this study four representative examples among the bright $\mathrm{O}$ stars and $\mathrm{B}$ Sgs for which we have been gathering spectroscopic time series during the last 4-6 yr as part of the IACOB project. We complement this sample of stars with another four representative targets selected from the study by Aerts et al. (2014) for purposes of comparison. A total of $\approx 30-170$ high-resolution spectra per target were analyzed in a homogeneous way, extracting information about $v \sin i, v_{\mathrm{mac}}$, and $R S k$ from each of the considered line profiles (O III $\lambda 5591$,

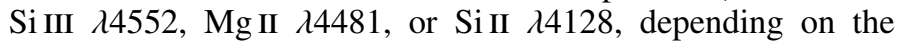
spectral type).

The list of targets considered for this study is presented in Table 3, where we also summarize the results from the time-dependent line-broadening analysis, the stellar parameters, and provide some notes about the type of variability.

The eight stars are located in the $v \sin i-v_{\mathrm{mac}}$ and sHR diagrams in Fig. 9. The interest and characteristics of these diagrams have been already described in previous sections. In this case, we incorporate information about the standard deviation and scatter resulting from the $v \sin i$ and $v_{\text {mac }}$ measurements of the complete time series into the $v \sin i-v_{\mathrm{mac}}$ diagram (thick and thin horizontal and vertical lines, respectively). We also overplot in the sHR diagram the instability domains for heat-driven $p$ and $g$ modes with $\ell=1$ (cyan and dark blue shaped regions, respectively) obtained by Godart et al. (2016), and the isocontours (red dashed lines) of the quantity $P_{\text {turb }}(\max ) / P$ computed by Grassitelli et al. (2015a) as described in Sect. 5.2.1.
Figure 10 illustrates the type of line-profile shape and variability associated with each star. This figure is complemented by Fig. 11, which shows the complete distribution of points in the $R S k-v \sin i / v_{\text {mac }}$ diagram resulting from the line-broadening and line-asymmetry analyses of the full spectroscopic dataset per target.

We highlight some interesting results from this study as follows:

- First, in O stars and B Sgs the scatter of the $v \sin i$ and $v_{\text {mac }}$ measurements resulting from the line-broadening analysis of the spectroscopic time series is not as extreme as in the pulsating or spotted B main-sequence stars (see Table 3). In particular, the standard deviation in the derived $v \sin i$ is always less than $10 \%$ in stars \#1-4, to be compared with the much higher values obtained for stars \#5-8. Therefore, the warning about the reliability of $v \sin i$ measurements highlighted by Aerts et al. (2014) is less critical for luminous OB stars, even though their line profiles have an important contribution of the macroturbulent broadening and show variability and asymmetries.

- A direct (qualitative) inspection of Fig. 10 already shows that while in all cases, sources of non-rotational broadening are clearly shaping the line profiles, the global shape and type of line variability is significantly different in both samples. Indeed, the occurrence of line-profile variability is most clearly detected by-eye in the pulsating or spotted B main-sequence stars and the considered B Sgs than in the two investigated O-type stars. In addition, the amount of variability is much larger in the B star sample, and the type of variability is qualitatively different.

- There are a variety of shapes and types of line-profile variability in the B sample; in contrast, these two characteristics are more homogeneous in the OB sample even though, as illustrated in the right panel of Fig. 9, this latter group of stars cover a wide range in evolutionary status and stellar properties.

- The amount of extra broadening is much larger in the $\mathrm{O}$ stars and B Sgs than in the pulsating or spotted B main-sequence stars (see Table 3 and left panel in Fig. 9).

- The spotted B star (HD 105382, \#7) is clearly separated in the $v_{\text {mac }} / v \sin i$ diagram (Fig. 9, left panel) from the rest of the considered targets. As indicated by Aerts et al. (2014) the spectroscopic variability produced by the spot can be mimicked in terms of a variable combination of $v \sin i$ and $v_{\text {mac }}$; however, the relative contribution of the non-rotational broadening is considerably smaller in this star (even the derived $v_{\text {mac }}$ ranges between $\approx 20$ and $40 \mathrm{~km} \mathrm{~s}^{-1}$ ).

- Among the pulsating B main-sequence stars, the star with a larger $v_{\text {mac }}$ and a profile shape that is closer to those found in the other group of stars is the non-radial, pressure-mode pulsator HD 111123 that is low-amplitude and multi-periodic (\#5, Aerts et al. 1998; Cuypers et al. 2002). However, the type of variability detected in this star is different from that observed in the other considered $\mathrm{O}$ stars and B Sgs. In particular, the variability is concentrated in the core of the line in the case of HD 111123, while in the OB stars the larger variability is detected in the wings.

- While the B main-sequence star identified as a non-radial gravity mode pulsator (HD 181558, \#8 De Cat \& Aerts 2002) is one of the candidates to show an important macroturbulent broadening contribution, the global broadening of the associated line profiles is much smaller compared to what is found in OB stars. However, this 
Table 3. Results from the time-dependent line-broadening analysis of a sample of eight $\mathrm{O}$ and $\mathrm{B}$ stars with an important contribution of non-rotational sources of line broadening.

\begin{tabular}{|c|c|c|c|c|c|c|c|c|c|c|c|c|}
\hline \multirow[t]{2}{*}{ \# } & \multirow[t]{2}{*}{ Star } & \multirow[t]{2}{*}{ Sp. C } & \multirow[t]{2}{*}{$N$} & \multicolumn{3}{|c|}{$v \sin i$} & \multicolumn{3}{|c|}{$v_{\mathrm{mac}}$} & \multirow[t]{2}{*}{$\log T_{\text {eff }}$} & \multirow[t]{2}{*}{$\log \mathscr{L} / \mathscr{L}_{\odot}$} & \multirow[t]{2}{*}{ Notes } \\
\hline & & & & $\bar{x}$ & $\sigma_{x}$ & Range & $\bar{x}$ & $\sigma_{x}$ & Range & & & \\
\hline \multicolumn{13}{|c|}{ O stars and B Sgs (OB stars) with multi-epoch observations gathered in the framework of the IACOB project } \\
\hline 1 & HD 199579 & O6.5 V((f))z & 112 & 56.2 & $8 \%$ & {$[48.0,69.0]$} & 81.2 & $7 \%$ & {$[65.0,90.4]$} & 4.60 & 3.83 & SB1 \\
\hline 2 & HD 36861 & O8 III & 172 & 54.2 & $3 \%$ & {$[49.4,60.0]$} & 73.2 & $4 \%$ & {$[64.3,80.0]$} & 4.55 & 4.06 & - \\
\hline 3 & HD 38771 & B0 Iab & 154 & 61.5 & $7 \%$ & {$[49.6,71.4]$} & 82.5 & $6 \%$ & {$[67.5,95.2]$} & 4.47 & 4.06 & - \\
\hline 4 & HD 34085 & B8 Iab & 108 & 38.7 & $7 \%$ & {$[32.3,47.6]$} & 52.9 & $10 \%$ & {$[47.1,67.5]$} & 4.10 & 4.10 & - \\
\hline \multicolumn{13}{|c|}{ B main-sequence stars from the study by Aerts et al. (2014) } \\
\hline 5 & HD 111123 & B0.5 IV & 119 & 28.6 & $22 \%$ & {$[16.5,41.9]$} & 42.8 & $11 \%$ & {$[33.1,57.3]$} & 4.43 & 3.42 & $\mathrm{SB} 1, \mathrm{nr}-\mathrm{p}$ \\
\hline 6 & HD 16582 & B2 IV & 59 & 11.6 & $23 \%$ & {$[4.1,16.1]$} & 17.7 & $10 \%$ & {$[12.7,20.8]$} & 4.36 & 3.04 & $\mathrm{r}$ \\
\hline 7 & HD 105382 & B6 III & 106 & 68.3 & $10 \%$ & {$[49.6,78.8]$} & 27.6 & $40 \%$ & {$[10.8,73.0]$} & 4.24 & 2.15 & spot \\
\hline 8 & HD 181558 & B5 III & 30 & 4.1 & $100 \%$ & {$[0,24.7]$} & 15.4 & $48 \%$ & {$[0.0,27.8]$} & 4.17 & 1.86 & nr-g \\
\hline
\end{tabular}
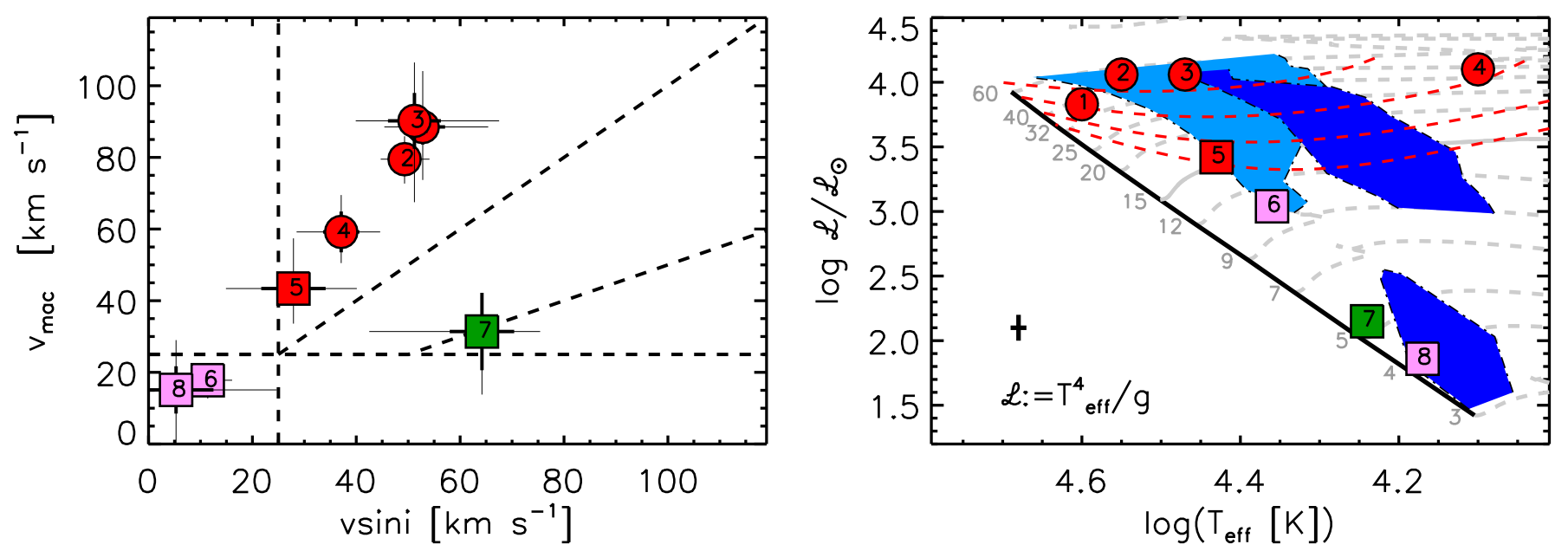

Fig. 9. Location of the 8 stars discussed in Sect. 5.2.3 in the $v \sin i-v_{\mathrm{mac}}$, and sHR diagrams. Same color code as is previous figures. The four pulsating or spotted B main-sequence stars considered by Aerts et al. (2014) are identified with square symbols, while the remaining OB stars surveyed by the IACOB project, with still unknown pulsational properties, are indicated with circles. The cyan and dark blue shaded regions in the sHR diagram indicate the instability domains for heat-driven $p$ and $g$ modes, respectively, with $\ell=1$ computed by Godart et al. (2016). The dashed red lines in the same figure indicate the isocontours of the quantity $P_{\text {turb }}(\max ) / P$ as computed by Grassitelli et al. (2015a).

characteristic may be explained by the fact that this star presents one dominant $g$ mode, while the collective effect of many modes is required to result in a much broadened line profile.

- There is a fairly good qualitative agreement between the predicted pulsational properties of the stars in terms of heat-driven modes, as indicated by the instability domains, and the observed line-profile variability ${ }^{5}$ (see right panel in Figs. 9 and 10, respectively). However, the connection between the global shape of the line profiles and the location of the associated stars inside or outside the instability domains is less clear or even non-existent. Contrarily, as indicated by Grassitelli et al. (2015a, see also Sect. 5.2.1), there seems to be a better correlation between the amount of extra broadening and the quantity $P_{\text {turb }}(\max ) / P$.

- All stars, except HD 199579 (\#1) and HD 36861 (\#2), show a considerable dispersion in the quantity $R S k$ (see Fig. 11). In the case of the pulsating or spotted B

\footnotetext{
5 Even though HD 34085 (Rigel) is located far outside the considered instability domains, the variability detected in this star has been proposed to be produced by the $\epsilon$ mechanism (Moravveji et al. 2012a,b).
}

main-sequence stars, the distribution of points around $R S k=0$ is roughly symmetric. The same occurs for HD 34085 (\#4). For HD 199579 (\#1), the distribution of points in the $R S k-v \sin i / v_{\text {mac }}$ diagram is compatible with a set of noisy (almost) symmetric line profiles (see also Fig. 10). Last, most of the analyzed line profiles in the spectroscopic time series of HD 36861 (\#2) and HD 38771 (\#3) have positive skewness.

This first combined investigation of line-broadening and line-profile variabilities in a small sample of $\mathrm{O}$ - and B-type stars highlights the need to incorporate time-series spectroscopy to study macroturbulent broadening, opening new sources of information for the understanding of this spectroscopic phenomenon (as already proposed by Aerts et al. 2014). The results highlighted above seems to lean in the same direction as the result indicated by the distribution of single snapshot line-broadening properties in the sHR diagram presented in Sect. 4.2.2 (see Fig. 5). Namely, a different origin of the extra source of non-rotational broadening in stars with masses above $\approx 15 M_{\odot}$ and $\mathrm{B}$ main-sequence stars. In addition, the inclusion of multi-epoch information seems to indicate that there is 

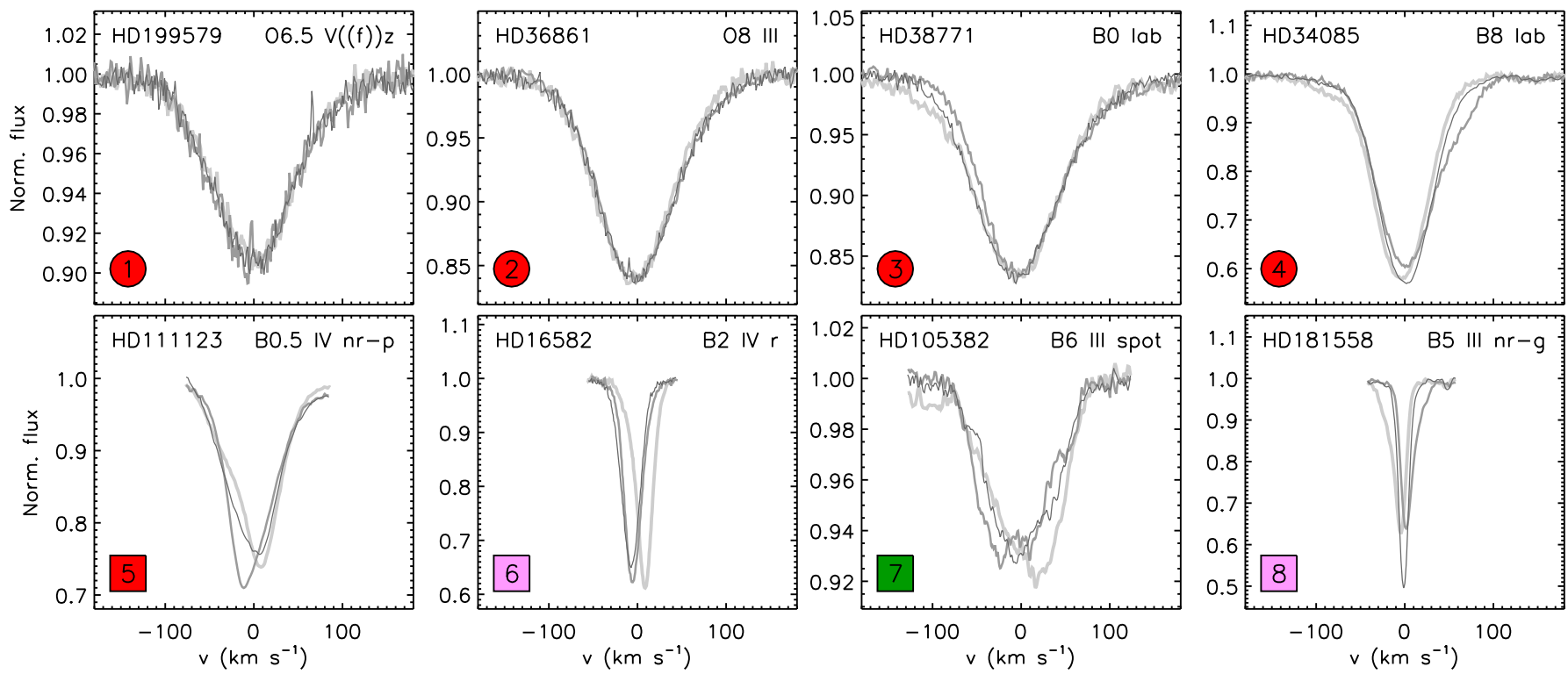

Fig. 10. Examples of line-profile shape and variability for the sample of stars quoted in Table 3 and discussed in Sect. 4. The three profiles shown in each panel correspond to different epochs in which the line-asymmetry analysis has resulted in the maximum, minimum, and mean values of $R S k$, respectively, as derived from the whole time series (see gray filled circles in Fig. 11). Note: HD 199579 and HD 111123 are two single line spectroscopic binaries; in both cases the line-profiles have been shifted in radial velocity to account for the orbital motion of the stars.
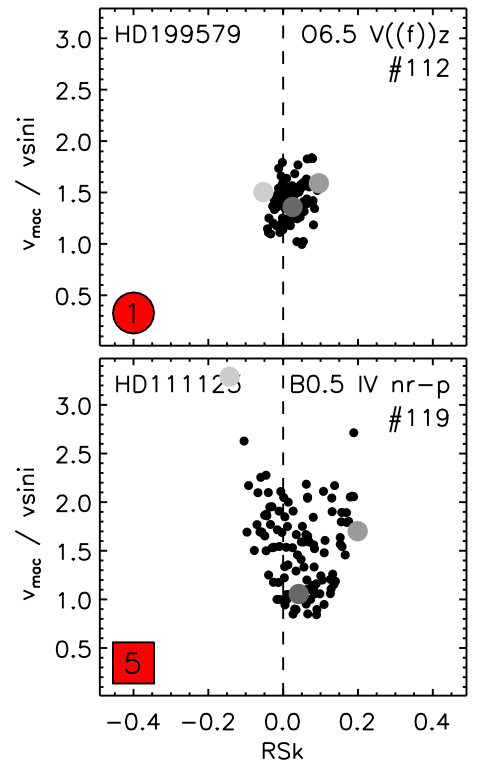
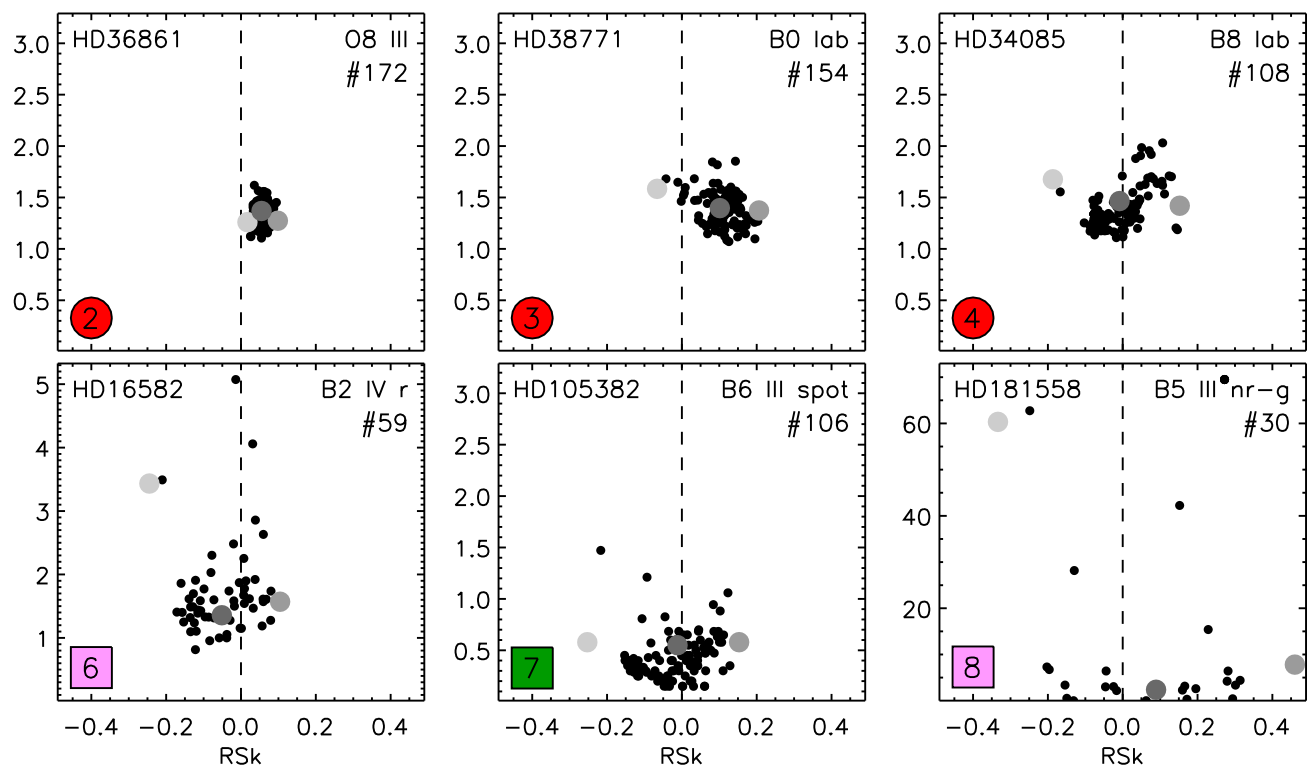

Fig. 11. Distribution of points in the $R S k-v \sin i / v_{\operatorname{mac}}$ diagram resulting from the line-broadening and line-asymmetry analyses of the full time series for the sample of stars quoted in Table 3. The three gray circles highlighted in each panel correspond to the line profiles shown in Fig. 10 (i.e., those profiles per target having minimum, maximum and close to the mean values of the quantity $R S k$ ). We note the different scale in the $y$-axis in each panel.

not necessarily a connection between the observed line-profile variability and the amount of line broadening.

\subsubsection{Concluding remarks}

In view of all the empirical clues presented in this paper and the various scenarios proposed to explain the occurrence of macroturbulent broadening in the OB star domain (see Sect. 5.2.1), we propose that the main observed properties of the line profiles of stars in the uppermost part of the sHR diagram are a result of the combined effect of (a) pulsation modes associated with a heat-driven mechanism and (b) possibly cyclic surface motions initiated by turbulent pressure instabilities.

Under this assumption, and combining the proposals by Aerts et al. (2009) and Grassitelli et al. (2015a), the latter mechanism would be mainly responsible for the large amount of non-rotational broadening detected in $\mathrm{O}$ stars and B Sgs, although further theoretical confirmation that the turbulent pressure scenario may end up in the observed broadening is still required. Then, operating at the same time in certain regions of the sHR diagram, long-lived $p$ and $g$ modes excited by the $\kappa$ or $\epsilon$ mechanisms (plus maybe other spectroscopic variability agents, such as spots, variable winds, or strange modes) would be the 
main agents producing the observed line-profile variability (and hence asymmetries).

As a consequence, above a certain $L / M$ ratio (or equivalently, a certain value of $\log \mathscr{L}$ ), stars with line profiles affected by a similar (dominating) macroturbulent broadening may present different type of line-profile variability depending on their location in the sHR diagram (as, e.g., predicted by the corresponding instability domains). Then, as the effect of turbulent pressure diminish when moving down toward the B star domain the observed line-profile characteristics are mainly produced by the effect of coherent heat-driven modes (or spots in certain cases).

This combined scenario could easily explain why, for example, HD 199579 (\#1) and HD 38771 (\#3) have very similar profiles in terms of global shape, but different types of variability. Indeed, no clear variability is detected in HD 199579 , as expected from the predicted absence of excited heat-driven modes for $\mathrm{O}$ stars close to the ZAMS, while the variability found in HD 38771 could be explained in terms of the predicted gravity-mode oscillations with a dominant tangential component. It would also explain why the profile of HD 111123 (\#5) is closer in shape to the line profiles commonly found in $\mathrm{O}$ stars and B Sgs because the star is located in a region of the sHR diagram where the effect of turbulent pressure begins to be important (see middle panel in Fig. 9). In addition, this type of line-profile variability is expected for a pressure-mode pulsator. Similar arguments could be applied to the remaining stars in the sample discussed in this section. For example, the remarkable line-profile variability (mainly concentrated in the wings, as expected from a $g$-mode pulsator), but low value of $v_{\text {mac }}$, associated with HD 181558 (\#8).

This is, hence, a scenario that allows us to explain most of the empirical evidence presented along this paper, and that must be further evaluated using time-dependent spectroscopy of a larger sample of targets (from the observational point of view), along with other theoretical simulations in the line of those presented in Aerts \& Rogers (2015).

\section{Summary and future prospects}

In this paper we provide new observational clues for the understanding of the empirical characteristics and physical origin of the so-called macroturbulent broadening in massive $\mathrm{O}$ and $\mathrm{B}$ stars. We base this study on a large, high-quality spectroscopic dataset compiled in the framework of the IACOB project during the last seven years. This observational material comprises high-resolution $(R=25000-85000)$, high signal-to-noise ratio $(S / N>150)$ spectra of a sample of 430 Galactic blue massive stars with spectral types in the range $\mathrm{O} 4-\mathrm{B} 9$ and covers all luminosity classes. In other words, we consider stars born with $M_{\text {ZAMS }}$ in the range $\approx 4-80 M_{\odot}$, and their evolved descendants up to effective temperatures on the order of $10000 \mathrm{~K}$. The sample can be roughly divided in $\mathrm{O}$ stars and $\mathrm{B} \mathrm{Sgs}$ (i.e., $\mathrm{OB}$ stars), which populate the uppermost part of the HR diagram, and B dwarfs and giants, which are located in or close to the main-sequence phase. We mainly concentrate in single snapshot spectra, but also consider available multi-epoch observations to identify and exclude clear spectroscopic binaries from the studied sample and to provide some empirical clues about the potential connection between macroturbulent broadening and line-profile variability.

We use a set of modern semi-automatic tools developed to perform quantitative spectroscopic analysis of large samples of $\mathrm{O}$ - and B-type stars in an objective, fast, and reliable way.
In this paper we concentrate on the information about the line-broadening $\left(v \sin i\right.$ and $v_{\text {mac }}$ ) and spectroscopic (mainly $T_{\text {eff }}$ and $\log g$ ) parameters extracted from the spectra. This information is complemented with the computation of specific quantities to quantify the amount of asymmetry $(R S k$, relative skewness) of the lines considered for the determination of the line-broadening parameters.

We identify a variety of line profiles in terms of shapes and broadening characteristics, which are quantified in terms of two line-broadening parameters. Following Simón-Díaz \& Herrero (2014), we divide the sample into two main groups (comprising three subgroups each) based on the ratio $v_{\text {mac }} / v \sin i$ and taking into account the limitations of the methodology considered for the determination of these parameters. The first group include stars showing an important (or dominant) macroturbulent broadening contribution to the line profile. The second group consist of stars in which either rotational broadening dominates or both $v \sin i$ and $v_{\text {mac }}$ are below the limits of reliability of the methodology. We also identify cases in which only upper limits of $v_{\text {mac }}$ can be determined. These mainly refer to line profiles dominated by rotational broadening. In this case, the derived values of $v_{\text {mac }}$ (which can indeed be large) must be considered with caution.

We then locate the complete sample in the spectroscopic HR diagram separating the stars by line-broadening characteristics. We find that the stars with an important contribution of macroturbulent broadening to their line profiles are mainly concentrated above $\approx 15 M_{\odot}$. From the distribution of line-broadening properties in the SHR diagram, we find empirical evidence suggesting the existence of various types of non-rotational broadening agents acting in the realm of massive stars. Even though all these additional sources of line-broadening could be quantitatively characterized in terms of $v_{\mathrm{mac}}$, and quoted as macroturbulent broadening, their physical origin can be actually different. Indeed, it is natural to think that some of them could be acting at the same time, with a different relative contribution to the line profiles, depending on the specific properties of the star at a given moment during its evolution. In particular, under this scenario, the distribution of stars in the sHR diagram indicates that, on the one hand, below $\approx 15 M_{\odot}$ (or, equivalently $\log \mathscr{L} / \mathscr{L}_{\odot} \approx 3.5$ ) the final shape of the stellar lines depends on a combination of different factors that are not only controlled by $T_{\text {eff }} \log g$, and/or $v \sin i$. On the other hand, the homogeneity in the type of profiles found in stars in the upper part of the sHR diagram $\left(\gtrsim 15 M_{\odot}\right)$ calls for one dominant broadening agent which, indeed, is operating in a very similar way in stars with similar $L / M$ but very different evolutionary stages.

The investigation of the distribution of single snapshot line-profile properties of our sample of $\mathrm{O}$ and $\mathrm{B}$ stars in the $R S k-v_{\text {mac }} / v \sin i$ diagram seems to indicate a potential connection between macroturbulent broadening, line asymmetries, and a variable phenomenon that is producing a time-dependent distribution of motions in the line formation region with an average velocity close to zero. This statement must, however, be confirmed using multi-epoch observations, since we have found that the effect of noise in the measurement of the quantity $R S k$ may also partly explain the observed distribution.

Following this idea of using multi-epoch observations to provide further observational clues to understand macroturbulent broadening in massive stars, a few years ago we started the compilation of a spectroscopic dataset specifically designed to this aim. While awaiting for the complete dataset, we present 
in this paper a proof-of-concept investigation of a sample of eight $\mathrm{O}$ and $\mathrm{B}$ stars for which we already count on long time-series observations gathered during several years, including $\approx 30-170$ spectra depending on the target. This sample comprises four O stars and B Sgs with still unknown pulsational properties (if any), and four of the well-known pulsating or spotted B main-sequence stars considered by Aerts et al. (2014). A preliminary comparative characterization of the global shape and type of variability found in this small sample of stars seems to also lean in the direction of a unique (or dominating) broadening agent acting in $\mathrm{O}$ stars and $\mathrm{B} \mathrm{Sgs}$, which may not be related to spots or the type of oscillations found in less massive B main-sequence stars (commonly associated with pressure and/or gravity heat-driven modes). In addition, we find observational evidence indicating that the line-broadening and line-profile variabilities observed in O stars and B Sgs may not be necessarily connected with the same physical driver.

Based on all the empirical results presented throughout this paper and the various scenarios proposed to date to explain the occurrence of macroturbulent broadening and line-profile variability in $\mathrm{O}$ and $\mathrm{B}$ stars, we launch the following hypotheses:

- Surface motions initiated by turbulent pressure instabilities generated in subsurface convection zones are mainly responsible for the dominant non-rotational, line-broadening component in $\mathrm{O}$ stars and B Sgs (as already pointed out by Grassitelli et al. 2015a).

- In the B main-sequence domain, once the effect of vigorous subsurface convection becomes negligible, stellar oscillations associated with heat-driven modes become important broadening agents (see also Aerts et al. 2014), especially when the projected rotational velocity of the star is low. However, these sources of line broadening never lead to values of $v_{\mathrm{mac}}$ as high as in the case of more massive stars.

- The most clearly detected line-profile variability is mainly produced by heat-driven pulsational modes, probably in combination with other mechanisms giving rise to spectroscopic variability, such as spots, wind variability, and strange modes.

All together, these ideas provide a scenario that explains most of the observational properties of the line profiles considered in this paper. However, before reaching a firm conclusion, it must still be further evaluated using (a) time-dependent spectroscopy of a larger sample of targets; (b) theoretical simulations to confirm that the turbulent pressure scenario may end up in the observed broadening; (c) results from the computation of instability domains of heat-driven high-degree modes for various assumptions on the considered opacities, metal mixture, overshooting parameter, or even on the stellar evolutionary code used as baseline for the adiabatic and non-adiabatic computations; and (d) an extension of the work by Aerts \& Rogers (2015) to the O star and B Sg domain.

In addition, the extension of the type of work presented here to other metallicities (e.g., considering a similar sample of stars in both Magellanic Clouds), and other stellar domains in the HR diagram (see, e.g., Grassitelli et al. 2015b) will certainly provide interesting clues to further support and dismiss scenarios about macroturbulent broadening in a more general context.

Acknowledgements. Based on observations made with the Nordic Optical Telescope, operated by NOTSA, and the Mercator Telescope, operated by the Flemish Community, both at the Observatorio del Roque de los Muchachos (La Palma, Spain) of the Instituto de Astrofísica de Canarias. This work has been funded by the Spanish Ministry of Economy and Competitiveness (MINECO) under the grants AYA2010-21697-C05-04, AYA2012-39364-C02-01, and Severo Ochoa SEV-2011-0187, and by the Research Council of KULeuven under grant GOA/2013/012. Part of this research received funding from the European Research Council under the European Community's H2020 Framework Programme, grant agreement No. 670519 (MAMSIE). This paper made use of the IAC Supercomputing facility HTCondor (http://research.cs . wisc. edu/htcondor/), recently expanded and improved thanks to FEDER funds granted by the Ministry of Economy and Competitiveness, project code IACA13-3E-2493. We thank Pieter Degroote for interesting discussions related to the work, and the anonymous referee for highlighting the most critical points of the first version of this paper.

\section{References}

Aerts, C., \& Rogers, T. M. 2015, ApJ, 806, L33

Aerts, C., de Pauw, M., \& Waelkens, C. 1992, A\&A, 266, 294

Aerts, C., De Cat, P., Cuypers, J., et al. 1998, A\&A, 329, 137

Aerts, C., Puls, J., Godart, M., \& Dupret, M.-A. 2009, A\&A, 508, 409

Aerts, C., Lefever, K., Baglin, A., et al. 2010a, A\&A, 513, L11

Aerts, C., Christensen-Dalsgaard, J., \& Kurtz, D. W. 2010b, in Asteroseismology, Astronomy and Astrophysics Library (Springer Science + Business Media B.V.)

Aerts, C., Simón-Díaz, S., Groot, P. J., \& Degroote, P. 2014, A\&A, 569, A118

Bailey, J. E., Nagayama, T., Loisel, G. P., et al. 2015, Nature, 517, 56B

Balona, L. A. 1986, MNRAS, 219, 111

Bouret, J.-C., Hillier, D. J., Lanz, T., \& Fullerton, A. W. 2012, A\&A, 544, A67

Briquet, M., Aerts, C., Lüftinger, T., et al. 2004, A\&A, 413, 273

Cantiello, M., Langer, N., Brott, I. et al. 2009, A\&A, 499, 279

Castro, N., Urbaneja, M. A., Herrero, A., et al. 2012, A\&A, 542, A79

Castro, N., Fossati, L., Langer, N., et al. 2014, A\&A, 570, L13

Conti, P. S., \& Ebbets, D. 1977, ApJ, 213, 438

Cuypers, J., Aerts, C., Buzasi, D., et al. 2002, A\&A, 392, 599

De Cat, P., \& Aerts, C. 2002, A\&A, 393, 965

Dufton, P. L., Ryans, R. S. I., Simón-Díaz, S., Trundle, C., \& Lennon, D. J. 2006, A\&A, 451, 603

Ekström, S., Georgy, C., Eggenberger, P., et al. 2012, A\&A, 537, A146

Fraser, M., Dufton, P. L., Hunter, I., \& Ryans, R. S. I. 2010, MNRAS, 404, 1306

Fossati, L., Castro, N., Schöller, M., et al. 2015, A\&A, 582, A45

Gray, D. F. 1976, in Research supported by the National Research Council of Canada (New York: Wiley-Interscience), 484

Gray, D. F. 1978, Sol. Phys., 59, 193

Grassitelli, L., Fossati, L., Simón-Diáz, S., et al. 2015a, ApJ, 808, L31

Grassitelli, L., Fossati, L., Langer, N., et al. 2015b, A\&A, 584, L2

Grevesse, N., \& Noels, A. 1993, in Perfectionnement de l'Association Vaudoise des Chercheurs en Physique, eds. B. Hauck, S. Paltani, \& D. Raboud, 205 Godart, M. 2011, Ph.D. Thesis, University of Liege, Belgium

Godart, M., Simón-Díaz, S., \& Herrero, A. 2016, A\&A, 597, A23 (Paper IV) Hekker, S., \& Aerts, C. 2010, A\&A, 515, A43

Howarth, I. D. 2004, in Stellar Rotation, Proc. IAU Symp. 215, ASP Conf. Ser., 33

Iglesias, C. A., \& Rogers, F. J. 1996, ApJ, 464, 943

Langer, N., \& Kudritzki, R. P. 2014, A\&A, 564, A52

Lefever, K., Puls, J., \& Aerts, C. 2007, A\&A, 463, 1093

Lefever, K., Puls, J., Morel, T., et al. 2010, A\&A, 515, A74

Lucy, L. B. 1976, ApJ, 206, 499

Markova, N., \& Puls, J. 2008, A\&A, 478, 823

Markova, N., Puls, J., Simón-Díaz, S., et al. 2014, A\&A, 562, A37

Martins, F., Hervé, A., Bouret, J.-C., et al. 2015, A\&A, 575, A34

Mahy, L., Rauw, G., De Becker, M., Eenens, P., \& Flores, C. A. 2015, A\&A, 577, A23

Martins, F., \& Palacios, A. 2013, A\&A, 560, A16

Miglio, A., Montalbán, J., \& Dupret, M.-A. 2007a, MNRAS, 375, L21

Miglio, A., Montalbán, J., \& Dupret, M.-A. 2007b, Comm. Asteroseismol., 151, 48

Moravveji, E. 2016, MNRAS, 455, L67

Moravveji, E., Guinan, E. F., Shultz, M., Williamson, M. H., \& Moya, A. 2012a, ApJ, 747, 108

Moravveji, E., Moya, A., \& Guinan, E. F. 2012b, ApJ, 749, 74

Noels, A., \& Scuflaire, R. 1986, A\&A, 161, 125

Pamyatnykh, A. A. 1999, Acta Astron., 49, 119

Puls, J., Urbaneja, M. A., Venero, R., et al. 2005, A\&A, 435, 669

Raskin, G., van Winckel, H., Hensberge, H., et al. 2011, A\&A, 526, A69

Reed, B. C. 2003, AJ, 125, 2531

Rivero González, J. G., Puls, J., Najarro, F., \& Brott, I. 2012, A\&A, 537, A79 
Rogers, T. M., Lin, D. N. C., McElwaine, J. N., \& Lau, H. H. B. 2013, ApJ, 772, 21

Ryans, R. S. I., Dufton, P. L., Rolleston, W. R. J., et al. 2002, MNRAS, 336, 577

Sabín-Sanjulián, C., Simón-Díaz, S., Herrero, A., et al. 2014, A\&A, 564, A39

Saio, H., Baker, N. H., \& Gautschy, A. 1998, MNRAS, 294, 622

Salmon, S., Montalbán, J., Morel, T. et al. 2012 MNRAS, 422, 3460

Samadi, R., Belkacem, K., Goupil, M. J., et al. 2010, Ap\&SS, 328, 253

Santolaya-Rey, A. E., Puls, J., \& Herrero, A. 1997, A\&A, 323, 488

Shiode, J. H., Quataert, E., Cantiello, M., \& Bildsten, L. 2013, MNRAS, 430, 1736

Schrijvers, C., Telting, J. H., Aerts, C., Ruymaekers, E., \& Henrichs, H. F. 1997, A\&AS, 121, 343

Simón-Díaz, S. 2015, IAU Symp., 307, 194

Simón-Díaz, S., \& Herrero, A. 2007, A\&A, 468, 1063

Simón-Díaz, S., \& Herrero, A. 2014, A\&A, 562, A135

Simón-Díaz, S., Herrero, A., Uytterhoeven, K., et al. 2010, ApJ, 720, L174

Simón-Díaz, S., Castro, N., Garcia, M., et al. 2011a, Bull. Soc. R. Sci. Liége, 80, 514
Simón-Díaz, S., Castro, N., Herrero, A., et al. 2011b, J. Phys. Conf. Ser., 328 , 012021

Simón-Díaz, S., Castro, N., Herrero, A., et al. 2012, Proc. Scientific Meeting in Honor of Anthony F. J. Moffat, 465, 19

Simón-Díaz, S., Negueruela, I., Maíz Apellániz, J., et al. 2015, Highlights of Spanish Astrophysics VIII, 576

Slettebak, A. 1956, ApJ, 124, 173

Sundqvist, J. O., Petit, V., Owocki, S. P., et al. 2013a, MNRAS, 433, 2497

Sundqvist, J. O., Simón-Díaz, S., Puls, J., \& Markova, N. 2013b, A\&A, 559, L10

Telting, J. H., \& Schrijvers, C. 1997, A\&A, 317, 723

Telting, J. H., Schrijvers, C., Ilyin, I. V., et al. 2006, A\&A, 452, 945

Telting, J. H., Ávila, G., Buchhave, L., et al. 2014, Astron. Nachr., 335, 41

Turck-Chièze, S., \& Gilles, D. 2013, EPJ Web Conf., 43, 01003

Unno, W., Osaki, Y., Ando, H., Saio, H., \& Shibahashi, H. 1989, in Nonradial Oscillations of Stars, 2nd edn. (Tokyo: University of Tokyo Press)

Ventura, P., D’Antona, F., \& Mazzitelli, I. 2008, Ap\&SS, 316, 93

Zdravkov, T., \& Pamyatnykh, A. A. 2008, J. Phys. Conf. Ser., 118, 012079

Wade, G. A., Grunhut, J., Alecian, E., et al. 2014, Magnetic Fields throughout Stellar Evolution, Proc. of IAU Symp., 302, 265 